\title{
Incidence and risk factors for important early morbidities associated with pediatric cardiac surgery in a UK population
}

\author{
Katherine L. Brown, MD, MPH, ${ }^{a}$ Deborah Ridout, MSc, ${ }^{b}$ Christina Pagel, $\mathrm{PhD},{ }^{\mathrm{c}}$ Jo Wray, PhD, \\ David Anderson, FRCS, ${ }^{\mathrm{d}}$ David J. Barron, MD, ${ }^{\mathrm{e}}$ Jane Cassidy, MRCP, ${ }^{\mathrm{e}}$ Peter J. Davis, FRCP, ${ }^{\mathrm{f}}$ \\ Warren Rodrigues, MRCP, ${ }^{\mathrm{g}}$ Serban Stoica, FRCS, ${ }^{\mathrm{f}}$ Shane Tibby, MRCP, ${ }^{\mathrm{d}}$ Martin Utley, $\mathrm{PhD},{ }^{\mathrm{c}}$ and \\ Victor T. Tsang, FRCS
}

\section{ABSTRACT}

Objective: Given excellent 30-day survival for pediatric cardiac surgery, other outcome measures are important. We aimed to study important early postoperative morbidities selected by stakeholders following a rigorous and evidencedbased process, with a view to identifying potential risk factors.

Methods: The incidence of selected morbidities was prospectively measured for 3090 consecutive pediatric cardiac surgical admissions in 5 UK centers between October 2015 and June 2017. The relationship between the candidate risk factors and the incidence of morbidities was explored using multiple regressions. Patient survival, a secondary outcome, was checked at 6 months.

Results: A total of $675(21.8 \%)$ procedure episodes led to at least 1 of the following: acute neurologic event, unplanned reoperation, feeding problems, renal replacement therapy, major adverse events, extracorporeal life support, necrotizing enterocolitis, surgical infection, or prolonged pleural effusion. The highest adjusted odds ratio of morbidity was in neonates compared with children, 5.26 (95\% confidence interval, 3.90-7.06), and complex heart diseases (eg, hypoplastic left heart), 2.14 (95\% confidence interval, 1.41-3.24) compared with low complexity (eg, atrial septal defect, $P<.001$ for all). Patients with any selected morbidity had a 6-month survival of $88.2 \%$ (95\% confidence interval, 85.490.6) compared with $99.3 \%$ (95\% confidence interval, 98.9-99.6) with no defined morbidity $(P<.001)$.

Conclusions: Evaluation of postoperative morbidity provides important information over and above 30-day survival and should become a focus for audit and quality improvement. Our results have been used to initiate UK-based audit for 5 of these 9 morbidities, co-develop software for local monitoring of these morbidities, and parent information about these morbidities. (J Thorac Cardiovasc Surg 2019;158:1185-96)

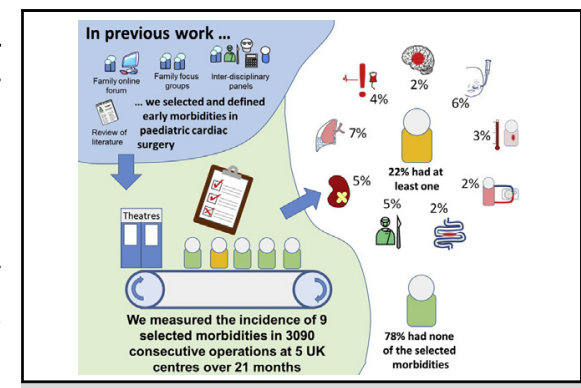

Study overview: selection of morbidities and prospective evaluation of morbidity incidence.

\section{Central Message}

The prospective evaluation of selected important early morbidities after pediatric cardiac surgery reveals a hidden burden over and above what is shown by the current key metric of early operative mortality.

\section{Perspective}

Early survival outcomes of pediatric cardiac surgery are excellent; thus, there is a strong interest from stakeholders to pursue a wider and more complex range of outcomes. Our prospective study in 3090 pediatric cardiac surgeries at 5 of the United Kingdom's 10 specialist centers indicated that standardized postoperative morbidity evaluation provides important data to supplement early survival assessment.

See Commentary on page 1197.

\footnotetext{
$\overline{\text { From the }{ }^{\mathrm{a}} \text { Cardiac }}$ and Critical Care Division, Great Ormond Street Hospital National Health Service Foundation Trust, Great Ormond Street, London, United Kingdom; ${ }^{\mathrm{b}}$ Population, Policy and Practice Programme, UCL Great Ormond Street Institute of Child Health, London, United Kingdom; 'Clinical Operational Research Unit, University College London, London, United Kingdom; ${ }^{\mathrm{d}}$ Department Paediatric Cardiology and Cardiac Surgery, Evelina London Children's Hospital, London, United Kingdom; ${ }^{\mathrm{e}}$ Department of Intensive Care and Paediatric Cardiac Surgery, Birmingham Children's Hospital, Birmingham, United Kingdom; ${ }^{\mathrm{f}}$ Paediatric Intensive Care Unit and Department of Paediatric Cardiac Surgery, Bristol Royal Children's Hospital, Bristol, United Kingdom; and ${ }^{\mathrm{g}}$ Department of Intensive Care and Paediatric Cardiac Surgery, Royal Hospital for Children, Glasgow, Scotland.

This project was funded by the National Institute for Health Research, Health Services and Delivery Research Program (Project No: 12/5005/06). K. Brown, D. Ridout, J. Wray, and V. Tsang were supported by the National Institute for Health Research Biomedical Research Centre at Great Ormond Street Hospital for Children NHS Foundation Trust and University College London.
}

The views and opinions expressed therein are those of the authors and do not necessarily reflect those of the NIHR HS\&DR program or the Department of Health.

Study registration: The study has ethical approval from London City Road Research Ethics Committee (14-LO-1442).

Read at the 99th Annual Meeting of The American Association for Thoracic Surgery, Toronto, Ontario, Canada, May 4-7, 2019.

Received for publication Nov 15, 2018; revisions received March 20, 2019; accepted for publication March 22, 2019; available ahead of print July 26, 2019.

Address for reprints: Katherine L. Brown, MD, MPH, Cardiac and Heart and Lung Division, Great Ormond Street Hospital NHS Foundation Trust, Great Ormond St, London WC1N 3JH, United Kingdom (E-mail: Katherine.brown@gosh.nhs. uk).

0022-5223

Copyright (C) 2019 by The American Association for Thoracic Surgery. Published by Elsevier Inc. This is an open access article under the CC BY-NC-ND license (http:// creativecommons.org/licenses/by-nc-nd/4.0/).

https://doi.org/10.1016/j.jtcvs.2019.03.139 


\section{Abbreviations and Acronyms}

CI = confidence interval

ECLS $=$ extracorporeal life support

NCHDA $=$ National Congenital Heart Diseases

Audit

$\mathrm{OR} \quad=$ odds ratio

$\mathrm{RR} \quad=$ risk ratio

$\mathrm{SD} \quad=$ standard deviation

\section{Scanning this QR code will take \\ (4) you to the article title page to access supplementary informa- tion. To view the AATS Annual Meeting Webcast, see the URL next to the webcast thumbnail.}

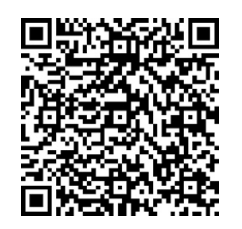

\section{INTRODUCTION}

\section{What Is Pediatric Cardiac Surgical Morbidity?}

Pediatric cardiac surgical morbidity is illness or lack of health that occurs soon after a cardiac operation, and so may be regarded as an adverse outcome of surgery. Although there has been considerable research on measuring, understanding, and reducing perioperative mortality, ${ }^{1-3}$ there has been less attention on surgical morbidities.

\section{Why Does Morbidity After Pediatric Cardiac Surgery Matter?}

Previous research on surgical morbidities after pediatric cardiac surgery has established their association with longer stays in hospital and other adverse outcomes, including death. ${ }^{4,5}$ For children with some heart conditions, prolonged postoperative stay in hospital is associated with higher levels of long-term neurologic disability. ${ }^{6}$ Prolonged hospitalization due to morbidities can be expensive to manage, for example, extracorporeal life support (ECLS) costs more than $£ 10,000$ per day. ${ }^{7}$ Morbidity, disability, and quality of life are viewed as key outcomes by patients, families, and clinical teams who are looking to deliver further improvements in service quality. In the United Kingdom, a recent major review of the specialty highlighted the need to monitor a range of outcomes including morbidity in a timely and meaningful fashion, ${ }^{8}$ and commissioners of services are appropriately seeking evidence on outcomes and quality assurance from providers.

\section{Our Study}

In previous work, a multidisciplinary group with patient and caregiver involvement selected a list of 9 key early postoperative morbidities ${ }^{9}$ that they considered most important, informed by clinical views on definitions and feasibility of routine monitoring. ${ }^{10}$ The selection process set out to identify the morbidities likely to have the greatest impact on patients in terms of hospital stay, mortality, quality, and cost (to be measured in a subset of patients and presented separately). Morbidities considered likely to have a lower impact or to be rare or difficult to reliably define and measure were not included.

The selected morbidities were ECLS, acute neurologic event, unplanned reintervention, feeding problems, major adverse event, prolonged pleural effusion, postsurgical infection morbidity, renal support, and necrotizing enterocolitis. A report detailing the definitions for each of these morbidities has been peer reviewed and published previously, ${ }^{10}$ and we include the main table from Brown and colleagues ${ }^{10}$ as Appendix E1. We report the incidence of and risk factors for these morbidities within the UK pediatric cardiac surgery population.

\section{MATERIALS AND METHODS Patient Population}

Our study population comprised all children aged less than 17 years undergoing cardiac surgery and open, closed, or hybrid procedures involving the heart as specified by the National Congenital Heart Disease Audit $(\mathrm{NCHDA})^{11}$ at each of 5 participating centers between October 1, 2015, and June 30, 2017, other than premature babies undergoing persistent ductus arteriosus ligation (who are mainly cared for in neonatal intensive care units) and children undergoing cardiothoracic transplant or tracheal procedures. These exclusions were made because these groups experience different sets of morbidities, and furthermore because of centralization of services in the United Kingdom, tracheal and transplant procedures are only carried out in one of the study sites. The participating centers care for approximately more than half of children with cardiac disease in the United Kingdom. ${ }^{11}$

\section{Data Collection}

Patients were prospectively monitored for the presence of the 9 early morbidities selected ${ }^{9}$ and defined ${ }^{10}$ in previous work as important and suitable for routine monitoring.

Data collection was undertaken prospectively, and morbidities were attributed to the immediately preceding cardiac surgery and defined within the same hospitalization other than unplanned reoperation within 30 days (an unanticipated cardiac procedure within 30 days was a morbidity outcome; Appendix E1,10 shows details) and mediastinitis (falls within postoperative infection morbidity, Brown and colleagues, ${ }^{10}$ and Appendix E1 shows details), both of which could be identified postdischarge by the operating surgeon and clinical care team.

As for the UK audit of 30 -day mortality, ${ }^{12}$ procedures on the same patient were included in the analysis of morbidity incidence if they occurred more than 30 days apart (ie, a new procedure occurring more than 30 days after the first or index operation was considered a separate clinical episode of care and included in the analysis as such). Planned operations within 30 days did not contribute to the analysis.

We obtained key clinical data on study patients from the local copy of $\mathrm{NCHDA}^{11}$ data held at each study site. All data were pseudonymized before sending them to the study team for analysis. The advantage of harnessing NCHDA data for this study was that each field is clearly and consistently defined. It is mandatory to record every cardiac procedure, and NCHDA data overall are externally validated. 
At the end of the study, we cross-checked the study population with NCHDA, removed operations that should have been excluded (eg, elective chest closures, miscoded interventional catheters), and verified all reoperation morbidities, including double-checking any ambiguous reoperations with the operating surgeon.

\section{Candidate Risk Factor Variables and Variable Groups}

We prespecified candidate clinical risk factors ${ }^{12,13}$ in the peer-reviewed study protocol. Most candidate risk factors were selected on the basis of previous known empiric association with mortality after pediatric cardiac surgery ${ }^{12-14}$ supplemented by a small number of candidate variables considered clinically important (sex, Down syndrome, prematurity).

The candidate risk factors considered in our analyses were sex, age band (neonate, infant, child), ${ }^{12}$ calculated weight-for-age $\mathrm{z}$-score, ${ }^{15}$ cardiac diagnosis category, functionally univentricular heart (yes/no), specific procedure type category, operation type (bypass, nonbypass, or hybrid), bypass time, acquired comorbidity, congenital comorbidity excluding Down syndrome, Down syndrome, additional cardiac risk factors, prematurity, and severity of illness indicator. ${ }^{12}$ We previously published the method by which the broad comorbidity groups that we used in this analysis were derived from 776 individual conditions. ${ }^{16}$ In this study, we used exactly the same comorbidity groups as in Brown and colleagues, ${ }^{16}$ and we summarize these in Table 1. For the variables of cardiac diagnosis and cardiac surgical procedure, we noted that there were many variables leading to some categories being sparsely populated. Therefore, these 2 variable groups were collapsed further to help with clinical interpretation. All subcategorizations were undertaken with reference to empiric data on risk of early mortality. ${ }^{1,12-14}$ These categorizations are summarized in Table 1, and details of how our previously published categories from Rogers and colleagues $^{12}$ were collapsed for this study of morbidity are provided in Appendix E2.

\section{Data Cleaning and Validation}

To ensure accuracy of study data and complete case ascertainment for incident morbidities, we took the following steps:

- A monthly telephone conference call involving at least 1 person from all sites discussed any ambiguous cases, and final case ascertainment was agreed.

- A 3-month sample of data from each study site (January 1, 2016, to March 31, 2016) was checked against an independent data source, NCHDA, for 5 of the morbidities.

- A final reconciliation of morbidities was undertaken at the end of the study when any cases with incomplete morbidity data were reviewed by the dedicated research nurse and a senior clinician at the sites.

\section{Sample Size}

In the original study protocol, we anticipated that between 3000 and 3300 surgical patients would be included across the 5 sites. ${ }^{11}$ On the basis of morbidity rates from a previous study, we calculated that this was sufficient to estimate accurately the incidence for morbidities occurring in at least $2 \%$ of cases. In the event the incidence in isolation was less than $1.5 \%$ for 5 of the morbidities, this meant that for analysis of risk factors for the primary morbidity outcome we needed to group morbidities for statistically robust analysis.

\section{Primary Outcome}

We used the following groupings of morbidity outcome for risk factor analysis:

- Two categories - any morbidity versus none of the selected morbidities, analyzed using multilevel logistic regression, accounting for multiple procedures within patients.

TABLE 1. Description of the approach to subcategorization of cardiac diagnosis, procedure, and comorbidity types for risk of morbidity modeling

\begin{tabular}{|c|c|}
\hline Risk factor category & Description of approach to subcategorization for risk modeling \\
\hline Cardiac diagnosis categories & $\begin{array}{l}\text { During the development of the PRAiS risk model }{ }^{12} \text { for } 30 \text {-d mortality after pediatric cardiac surgery, } 28 \\
\text { cardiac diagnosis categories were ranked by an expert panel based on a combination of both complexity } \\
\text { and empirically derived risk of death, with the described independent validation of model performance } \\
\text { in a test dataset. } \\
\text { To develop models with risk factors for the outcome of morbidity, we further collapsed these } 28 \text { cardiac } \\
\text { diagnoses into } 5 \text { groups, ranked by complexity and risk of death in the original study }{ }^{12} \text { from A (most } \\
\text { severe, eg, hypoplastic left heart syndrome) to E (least severe, eg, atrial septal defect) (Appendix E2). }\end{array}$ \\
\hline Specific cardiac procedure categories & $\begin{array}{l}\text { NCHDA developed an algorithm for grouping pediatric cardiac operations into relatively homogeneous } \\
\text { procedure categories for reporting mortality outcomes. }{ }^{11} \\
\text { To develop models with risk factors for the outcome of morbidity, we collapsed these } 50 \text { procedure groups } \\
\text { (includes not a procedure grouping) into } 3 \text { broad categories of reparative or corrective operation, } \\
\text { palliative or staging operation, and ungrouped operation (where the approach could not be determined) } \\
\text { (Appendix E2). }\end{array}$ \\
\hline Comorbidity categories & $\begin{array}{l}\text { To develop models with risk factors for the outcome of morbidity, we included the preexisting comorbidity } \\
\text { groups developed for pediatric cardiac procedures }{ }^{16} \text { that appear as independent risk factors for mortality } \\
\text { in the UK-based risk adjustment model for } 30 \text {-d mortality after pediatric cardiac surgery. }{ }^{12} \\
\text { These are (1) acquired comorbidity (eg, renal failure, stroke), (2) congenital comorbidity excluding Down } \\
\text { syndrome (eg, congenital defect of a major organ or genetic syndrome), (3) additional cardiac risk } \\
\text { factors (eg, cardiomyopathy, pulmonary hypertension), and (4) severity of illness indicator (eg, } \\
\text { preprocedure respiratory failure or shock). We also included for consideration of any link to morbidity } \\
\text { (5) Down syndrome and (6) prematurity (gestational age }<37 \mathrm{wk} \text { ), although they were not statistically } \\
\text { linked to postoperative mortality. }{ }^{12}\end{array}$ \\
\hline
\end{tabular}

PRAiS, Partial Risk Adjustment in Surgery risk model (the UK-based method of risk stratification for early postoperative mortality); NCHDA, National Congenital Heart Diseases Audit. 
- Four categories-no selected morbidity, single selected morbidity other than ECLS; multiple morbidity with no ECLS; and ECLS. This grouping of outcomes enables the discrimination of risk factors for the particularly adverse outcomes of ECLS and multiple morbidities as identified a priori. ${ }^{17-21}$

\section{Analysis of Risk Factors for Primary Outcome}

The prevalence of candidate risk factors is described with frequency (\%) for categoric factors and mean (standard deviation [SD]) or median (interquartile range) as appropriate for continuous factors. For the outcome of any selected morbidity versus no selected morbidity, the estimated effects are presented as odds ratios (ORs) and 95\% confidence intervals (CIs).

For the 4 category morbidity outcomes, we used multinomial logistic regression with robust standard errors to adjust for clustering within patients. Estimated effects are presented as risk ratios (RRs) and 95\% CI. For both outcome groupings, we investigated whether the inclusion of site as a random factor was important.

For both outcome groupings, univariate models were fitted for each of the prespecified candidate risk factors, and the estimated effects of the factors on morbidity outcome are presented along with $95 \%$ CIs. All factors significant on univariate analysis $(P<.1)$ were included in the multivariable models. We state the number of missing values where relevant in results. We used multiple imputation by chained equations to account for missing data, and the imputation model included all risk factors considered in the univariate analysis, which we assumed to include all predictors of whether a data item would be missing. The final multivariable models were derived by fitting a regression model for all significant predictors, and estimates were combined using $\mathrm{Ru}$ bin's rules. ${ }^{22}$ Model performance for the final multivariable models was assessed using the c-statistic (area under the receiver operator curve) and Hosmer-Lemeshow statistic. All analyses were performed in Stata $14 .^{23}$

\section{Secondary Outcomes}

The life status of patients in the study at 6 months after each operation was determined using a combination of hospital records and NCHDA data in March 2018. Thirty-day and 6-month mortality were attributed to the first appearance of each patient in the dataset.
Length of stay was defined as the number of whole days between the operation that led to the child entering the study and the date of discharge from the specialist cardiac center. Two data sources (study database and NCHDA) were cross-checked for accuracy.

Mortality within 6 months of each patient's first procedure was compared between patients with and without a morbidity using logistic regression.

\section{RESULTS \\ Descriptive Data}

After removal of 10 misclassified procedures (minor and excluded procedures, such as chest reopenings), 63 cardiac operations that were undertaken within 30 days as part of the planned treatment pathway, and all unplanned reoperations within 30 days (a morbidity outcome, Table 2, there were 161 in total), there were 3090 procedures meeting inclusion/exclusion criteria. These procedures pertained to 2861 patients, of whom 2648 had 1 surgical procedure, 197 had 2 surgical procedures, and 16 had 3 surgical procedures.

Of the 3090 procedures included in the study, 1723 $(56 \%)$ were reparative, $510(16 \%)$ were palliative, and $857(28 \%)$ were ungrouped. Of these procedure episodes, $414(13.4 \%)$ were in functionally univentricular circulations, $528(17.1 \%)$ were in neonates, $1291(41.8 \%)$ were in infants, and $1271(41.3 \%)$ were in children.

There was excellent concurrence between the study data set and NCHDA; among the 443 patients in the 3-month sample checked, 9 morbidities were present in the study dataset but not in NCHDA, and in no cases was a morbidity recorded in NCHDA and not in the study dataset.

\section{Incidence of Morbidities}

We present the incidence of individual selected morbidities as "any occurrence" (the total number of occurrences

TABLE 2. Number and percentage of procedure episodes affected by the various types of morbidity

\begin{tabular}{|c|c|c|}
\hline & $\begin{array}{c}\text { Of all } 3090 \text { procedures, the number } \\
(\%[95 \% \mathrm{CI}]) \text { that had had the stated morbidity, } \\
\text { inclusive of combined morbidities and } \\
\text { morbidities in isolation }\end{array}$ & $\begin{array}{c}\text { Of all } 3090 \text { procedures, the number } \\
(\%[95 \% \mathrm{CI}]) \text { that had the stated morbidity } \\
\text { by itself as an isolated event }\end{array}$ \\
\hline Any morbidity & $675(21.8 \%)$ & Not applicable \\
\hline Multiple morbidity & $197(6.4 \%$ [5.5-7.3]) & Not applicable \\
\hline ECLS & $62(2 \%[1.5-2.6])$ & $6(0.2 \%)$ \\
\hline Prolonged pleural effusion & $202(6.5 \%$ [5.7-7.5]) & $111(3.6 \%[3.0-4.3])$ \\
\hline Feeding problems & $184(6.0 \%$ [5.1-6.8]) & $99(3.2 \%$ [2.6-3.9]) \\
\hline Unplanned reintervention & $161(5.2 \%[4.5-6.1])$ & $59(1.9 \%$ [1.5-2.5]) \\
\hline Renal support & $143(4.6 \%$ [3.9-5.4]) & $40(1.3 \%$ [0.9-1.8]) \\
\hline Major adverse event & $134(4.3 \%$ [3.6-5.1]) & $34(1.1 \%$ [0.8-1.5]) \\
\hline Postsurgical infection & $85(2.8 \%$ [2.2-3.4]) & $27(0.9 \%$ [0.6-1.3]) \\
\hline Necrotizing enterocolitis & $75(2.4 \%$ [1.9-3.0]) & $32(1.0 \%[0.7-1.5])$ \\
\hline Acute neurologic event & $66(2.1 \%[1.7-2.7])$ & $14(0.5 \%[0.2-0.8])$ \\
\hline
\end{tabular}

CI, Confidence interval; ECLS, extracorporeal life support. 
of a given morbidity both as part of a multi-morbidity and as a standalone event) and "in isolation" (where the morbidity occurred as a stand-alone event) in Figure 1 and Table 2. Given that we had an a priori interest in ECLS as a severe adverse event, when ECLS occurred, patients were defined as ECLS morbidity irrespective of other concurrent morbidities. The most common morbidities as "any occurrence" were prolonged pleural effusion $(6.5 \%)$, feeding problems $(6.0 \%)$, and unplanned reintervention $(5.2 \%)$. We prespecified in the protocol that morbidities with a rate less than $1.5 \%$ could be considered rare. All of the 9 selected morbidities had any occurrence rate greater than $1.5 \%$, the least common being an acute neurologic event $(2.1 \%)$. However, only 4 morbidities occurred in isolation at a rate greater than $1.5 \%$ : prolonged pleural effusion, feeding problems, unplanned reintervention, and ECLS.

\section{Multiple Morbidities}

Of $197(6.4 \%)$ procedures that resulted in multiple morbidity, $76(39 \%)$ were with a feeding problem, 73 $(37 \%)$ with an unplanned reintervention, $72(37 \%)$ with prolonged pleural effusion, 67 (34\%) with major adverse event, $66(34 \%)$ with renal support, $49(25 \%)$ with postsurgical infection, $34(17 \%)$ with acute neurologic event, and
$33(17 \%)$ with necrotizing enterocolitis. For the 197 multiple morbidity cases, 140 involved 2 morbidities, 39 involved 3 morbidities, 17 involved 4 morbidities, and 1 involved 5 morbidities.

\section{ECLS Morbidities}

Among the $62(2 \%)$ procedures in which there was postoperative ECLS, only 6 involved just ECLS and no other morbidities, $37(60 \%)$ ECLS morbidities involved renal support; $33(53 \%)$ were with major adverse event, $29(47 \%)$ were with unplanned reintervention, $19(31 \%)$ were with prolonged pleural effusion, $16(29 \%)$ were with an acute neurologic event, 10 $(16 \%)$ were with necrotizing enterocolitis, $9(15 \%)$ were with postsurgical infection, and $9(15 \%)$ were with a feeding problem.

\section{Risk Factors for Occurrence of Any Morbidity Versus No Morbidity}

Table 3 shows the frequency (\%) for categoric risk factors and mean (SD) or median (interquartile range) as appropriate for continuous risk factors. Weight was missing or infeasible ( $>5 \mathrm{SD}$ from the normative mean) in $186 \mathrm{pa}-$ tients, and for these we used multiple imputation to infer their weight.

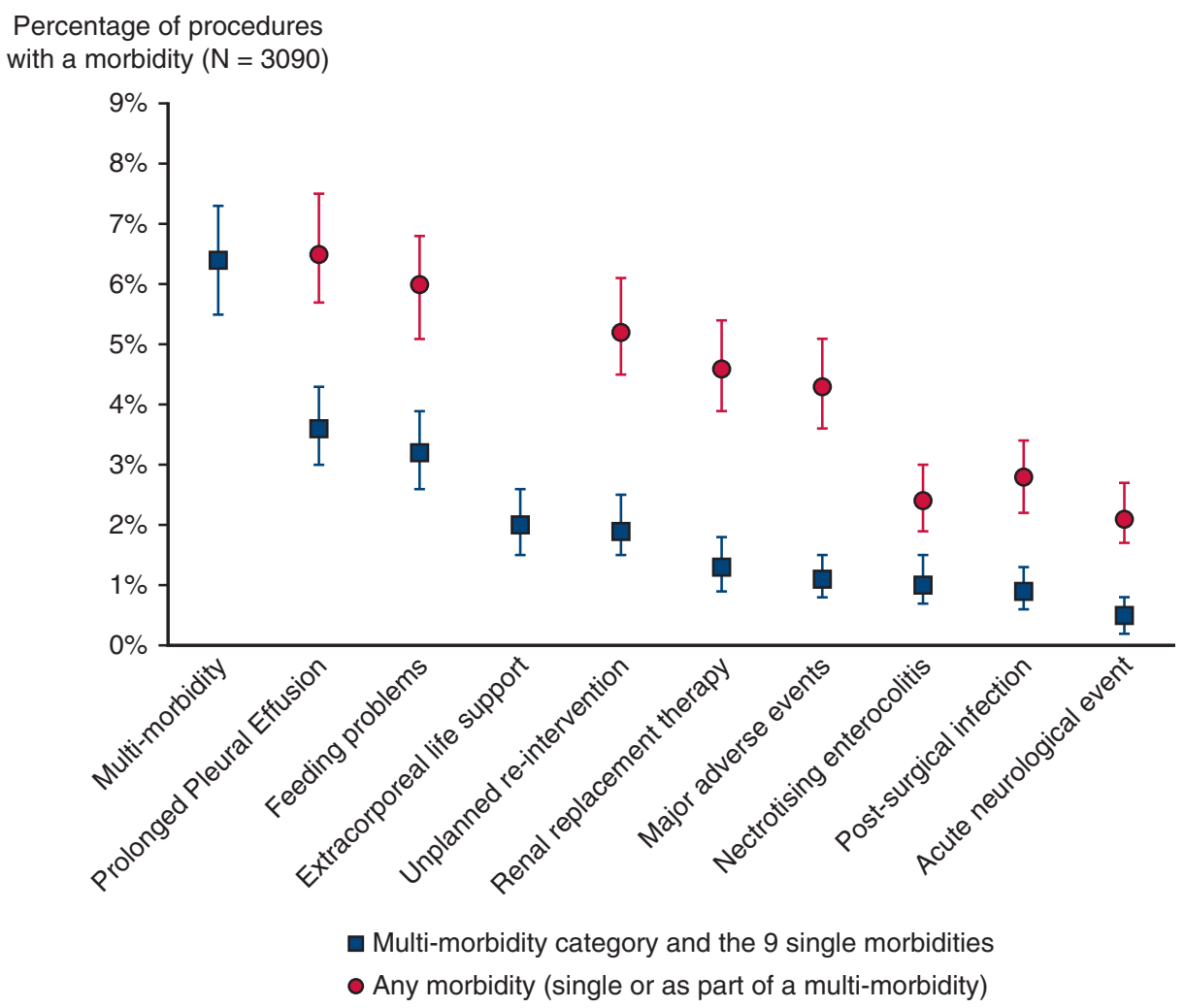

FIGURE 1. Incidence of selected morbidities by procedure with $95 \%$ CIs. 1, In red, all instances of each of the defined morbidities including as single morbidities, as part of multi-morbidities and as part of ECLS. 2, In blue, the numbers for each of the defined morbidities when these occurred in isolation. 
TABLE 3. Summary of risk factors by any morbidity outcome, with univariate and multivariable logistic regression results

\begin{tabular}{|c|c|c|c|c|}
\hline & $\begin{array}{l}\text { No morbidity } \\
(\mathrm{N}=\mathbf{2 4 1 5})\end{array}$ & $\begin{array}{l}\text { Any morbidity } \\
\qquad(N=675)\end{array}$ & $\begin{array}{c}\text { Univariate } O R \\
(95 \% \text { CI }) P \text { value }\end{array}$ & $\begin{array}{c}\text { Multivariable } \\
\text { OR }(95 \% \text { CI }) P \text { value }\end{array}$ \\
\hline Male & $1299(53.8)$ & $372(55.1)$ & $1.05(0.89-1.25) .54$ & - \\
\hline Median age (d), (IQR) & $286(105-1582)$ & $102(10-331)$ & & \\
\hline Child (ref) & $1111(46.0)$ & $160(23.7)$ & & \\
\hline Infant & $1023(42.4)$ & $268(39.7)$ & $1.82(1.47-2.25)<.001$ & $1.61(1.26-2.05)<.001$ \\
\hline Neonate & $281(11.6)$ & $247(36.6)$ & $6.10(4.81-7.75)<.001$ & $5.26(3.90-7.09)<.001$ \\
\hline Median weight (kg), (IQR) & $7.7(4.7-16.2)$ & $4.6(3.2-8.0)$ & & \\
\hline Weight $<$ mean for age -2 SD & $714(31.5)$ & $234(36.8)$ & $1.27(1.05-1.52) .01$ & $1.21(0.97-1.51) .09$ \\
\hline \multicolumn{5}{|l|}{ Primary cardiac diagnosis } \\
\hline E (ref)-least severe/complex disease & $1002(41.5)$ & $123(18.2)$ & & \\
\hline $\mathrm{D}$ & $796(33.0)$ & 227 (33.6) & $5.13(3.79-6.93)<.001$ & $2.02(1.58-2.60)<.001$ \\
\hline $\mathrm{C}$ & $215(8.9)$ & $109(16.2)$ & $3.83(2.85-5.13)<.001$ & $1.44(1.00-2.07) .05$ \\
\hline B & $232(9.6)$ & $109(16.2)$ & $4.13(3.07-5.55)<.001$ & $2.62(1.85-3.71)<.001$ \\
\hline A-most severe/complex disease & $170(7.0)$ & $107(15.8)$ & $2.32(1.83-2.94)<.001$ & $2.14(1.41-3.24)<.001$ \\
\hline Univentricular heart & $255(10.6)$ & 159 (23.6) & $2.61(2.11-3.23)<.001$ & $1.55(1.07-2.24) .02$ \\
\hline Acquired comorbidity & $337(14.0)$ & $119(17.6)$ & $1.32(1.05-1.66) .02$ & $1.33(1.03-1.71) .03$ \\
\hline Congenital comorbidity & $537(22.2)$ & $178(26.4)$ & $1.25(1.03-1.52) .03$ & $1.28(1.02-1.59) .03$ \\
\hline Severity of illness risk & $222(9.2)$ & $152(22.5)$ & $2.87(2.30-3.58)<.001$ & $1.52(1.16-2.00)<.01$ \\
\hline Premature birth & $231(9.6)$ & $73(10.8)$ & $1.15(0.87-1.51) .33$ & - \\
\hline Downs syndrome & $214(8.9)$ & $63(9.3)$ & $1.06(0.79-1.43) .71$ & - \\
\hline Additional cardiac risk factors & $165(6.8)$ & $65(9.6)$ & $1.45(1.09-1.94) .01$ & $1.39(0.99-1.94) .05$ \\
\hline Procedure reparative/corrective (ref) & $1391(57.6)$ & $332(49.2)$ & & \\
\hline Palliative/staged & $331(13.7)$ & $179(26.5)$ & $2.27(1.82-2.82)<.001$ & $1.65(1.14-2.38)<.01$ \\
\hline Ungrouped or ambiguous & $693(28.7)$ & $164(24.3)$ & $0.99(0.81-1.22) .94$ & $1.04(0.82-1.31) .75$ \\
\hline Median bypass time (min) (IQR) & $72(42-110)$ & $110(62-156)$ & & \\
\hline No bypass (ref) & $390(16.2)$ & $103(15.3)$ & & \\
\hline Up to $90 \mathrm{~min}$ & $1148(47.5)$ & $150(22.2)$ & $0.48(0.35-0.65)<.001$ & $0.78(0.57-1.09) .14$ \\
\hline$>90 \mathrm{~min}$ & $877(36.3)$ & $422(62.5)$ & $1.76(1.32-2.34)<.001$ & $2.28(1.67-3.12)<.001$ \\
\hline
\end{tabular}

Cardiac diagnosis group (main preoperative diagnosis) (A) hypoplastic left heart syndrome, truncus arteriosus, pulmonary atresia intact septum, (B) functionally univentricular heart, pulmonary atresia ventricular septal defect, (C) transposition of the great arteries all types, interrupted aortic arch, totally anomalous pulmonary venous connection, (D) patent ductus arteriosus, tricuspid valve anomalies, acquired heart disease, complete atrioventricular septal defect, (E) tetralogy of Fallot, mitral valve anomalies, isolated aortic stenosis, aortic regurgitation, aortic arch obstruction, subaortic obstruction, ventricular septal defect, atrial septal defect. OR, Odds ratio; $C I$, confidence interval; IQR, interquartile range; $S D$, standard deviation.

With the exception of patient sex, prematurity, Down syndrome, and low weight for age, all candidate risk factors were statistically associated with any morbidity outcome in multivariable analysis (Table 3 shows ORs and 95\% CIs). Inclusion of site made a negligible difference, and we present results from the model without site. After adjustment for other factors, age was the most important risk factor: $\mathrm{Ne}$ onates had a 5.26-fold increased chance of morbidity, and infants had a 1.61-fold increased risk compared with children aged more than 1 year. Cardiac diagnosis group was the next most influential factor, with the more complex conditions carrying a higher risk of morbidity, followed by a prolonged bypass time in excess of 90 minutes, which carried a 2.8-fold increased risk. A palliative or staged procedure and the presence of a functionally univentricular heart both increased the chance of a morbidity 1.6 times (these 2 factors clearly have some overlap). Severity of illness factor (which includes preprocedure mechanical ventilation or shock) increased the risk by 1.5 times.

The area under the receiver operator curve for the final multiple logistic regression model for any morbidity was 0.77 (95\% CI, 0.75-0.79), and Hosmer-Lemeshow goodness of fit was $P=.13$, indicating reasonable calibration of the model.

\section{Risk Factors for the 4-Level Morbidity Outcome}

The multinomial models for the 4-category outcome are shown in Table 4 . These analyses were in line with the 
TABLE 4. Multinomial regression results of risk factors for the 4-level morbidity outcome

\begin{tabular}{|c|c|c|c|c|c|c|}
\hline & $\begin{array}{c}\text { Univariate RR } \\
\text { single vs none } \\
(95 \% \mathrm{CI}) \\
P \text { value }\end{array}$ & $\begin{array}{c}\text { Univariate RR } \\
\text { ECLS vs none } \\
(95 \% \text { CI }) \\
P \text { value }\end{array}$ & $\begin{array}{c}\text { Univariate } \\
\text { RR multiple vs none } \\
(95 \% \text { CI }) \\
P \text { value }\end{array}$ & $\begin{array}{c}\text { Multivariable RR } \\
\text { single vs none } \\
(95 \% \mathrm{CI}) \\
P \text { value }\end{array}$ & $\begin{array}{c}\text { Multivariable RR } \\
\text { ECLS vs none } \\
(95 \% \text { CI }) \\
P \text { value }\end{array}$ & $\begin{array}{c}\text { Multivariable } \\
\text { RR multi vs none } \\
(95 \% \mathrm{CI}) \\
P \text { value }\end{array}$ \\
\hline Male & $\begin{array}{c}1.19(0.97-1.48) \\
.10\end{array}$ & $\begin{array}{c}0.62(0.37-1.03) \\
.07\end{array}$ & $\begin{array}{c}0.96(0.72-1.29) \\
.79\end{array}$ & - & - & - \\
\hline \multicolumn{7}{|l|}{ Child (ref) } \\
\hline Neonate & $\begin{array}{c}1.66 \\
(1.29-2.13) \\
<.001 \\
4.44 \\
(3.34-5.90) \\
<.001\end{array}$ & $\begin{array}{c}2.27 \\
(1.10-4.68) \\
.03 \\
10.06 \\
(4.95-20.46) \\
<.001\end{array}$ & $\begin{array}{c}2.20 \\
(1.46-3.33) \\
<.001 \\
10.28 \\
(6.81-15.51) \\
<.001\end{array}$ & $\begin{array}{c}1.49 \\
(1.12-1.97) \\
<.01 \\
3.79 \\
(2.71-5.30) \\
<.001\end{array}$ & $\begin{array}{c}2.00 \\
(0.90-4.44) \\
.09 \\
7.47 \\
(2.94-18.94) \\
<.001\end{array}$ & $\begin{array}{c}1.88 \\
(1.21-2.92) \\
<.01 \\
10.52 \\
(6.22-17.78) \\
<.001\end{array}$ \\
\hline $\begin{array}{c}\text { Weight }<\text { mean for } \\
\text { age }-2 \mathrm{SD}\end{array}$ & $\begin{array}{c}1.25(1.00-1.57) \\
.05\end{array}$ & $\begin{array}{c}1.09(0.63-1.87) \\
.76\end{array}$ & $\begin{array}{c}1.36(0.99-1.85) \\
.05\end{array}$ & $\begin{array}{c}1.23(0.94-1.60) \\
.13\end{array}$ & $\begin{array}{c}0.98(0.51-1.88) \\
.95\end{array}$ & $\begin{array}{c}1.26(0.88-1.80) \\
.21\end{array}$ \\
\hline \multicolumn{7}{|l|}{$\begin{array}{c}\text { Cardiac diagnosis } \\
\text { E (ref-least seve }\end{array}$} \\
\hline $\mathrm{D}$ & $\begin{array}{l}1.88(1.42-2.49) \\
<.001\end{array}$ & $\begin{array}{c}4.20(1.68-10.50) \\
<.01\end{array}$ & $\begin{array}{c}3.57(2.22-5.73) \\
<.001\end{array}$ & $\begin{array}{c}1.63(1.22-2.18) \\
<.01\end{array}$ & $\begin{array}{c}3.12(1.24-7.87) \\
.02\end{array}$ & $\begin{array}{l}3.50(2.14-5.72) \\
\quad<.001\end{array}$ \\
\hline $\mathrm{C}$ & $\begin{array}{l}2.96(2.07-4.23) \\
\quad<.001\end{array}$ & $\begin{array}{c}12.43(4.81-32.13) \\
<.001\end{array}$ & $\begin{aligned} 6.60 & (3.84-11.36) \\
& <.001\end{aligned}$ & $\begin{array}{c}1.27(0.85-1.92) \\
.25\end{array}$ & $\begin{array}{c}2.20(0.68-7.14) \\
.19\end{array}$ & $\begin{array}{c}1.87(0.96-3.62) \\
.06\end{array}$ \\
\hline B & $\begin{array}{c}2.93(2.06-4.15) \\
<.001\end{array}$ & $\begin{aligned} 8.64 & (3.21-23.25) \\
& <.001\end{aligned}$ & $\begin{array}{c}6.12(3.56-10.52) \\
<.001\end{array}$ & $\begin{array}{c}1.88(1.24-2.87) \\
<.01\end{array}$ & $\begin{aligned} & 8.53(3.01-24.19) \\
&<.001\end{aligned}$ & $\begin{aligned} 4.54 & (2.44-8.46) \\
& <.001\end{aligned}$ \\
\hline A (most severe) & $\begin{array}{l}3.93(2.74-5.63) \\
\quad<.001\end{array}$ & $\begin{array}{l}7.86(2.69-22.93) \\
\quad<.001\end{array}$ & $\begin{array}{c}9.09(5.30-15.57) \\
\quad<.001\end{array}$ & $\begin{array}{c}1.67(1.03-2.71) \\
.04\end{array}$ & $\begin{array}{c}4.29(1.11-16.55) \\
.03\end{array}$ & $\begin{array}{c}3.50(1.71-7.16) \\
\quad<.01\end{array}$ \\
\hline Univentricular heart & $\begin{array}{l}2.61(2.02-3.37) \\
\quad<.001\end{array}$ & $\begin{array}{c}1.44(0.70-2.94) \\
.32\end{array}$ & $\begin{array}{c}3.04(2.15-4.29) \\
<.001\end{array}$ & $\begin{array}{c}1.54(1.03-2.32) \\
.04\end{array}$ & $\begin{array}{c}1.13(0.35-3.63) \\
.84\end{array}$ & $\begin{array}{c}1.80(0.97-3.34) \\
.06\end{array}$ \\
\hline $\begin{array}{l}\text { Acquired } \\
\text { comorbidity }\end{array}$ & $\begin{array}{c}1.27(0.95-1.69) \\
.10\end{array}$ & $\begin{array}{c}1.33(0.69-2.57) \\
.40\end{array}$ & $\begin{array}{c}1.43(0.98-2.07) \\
.06\end{array}$ & $\begin{array}{c}1.31(0.97-1.77) \\
.08\end{array}$ & $\begin{array}{c}1.15(0.55-2.40) \\
.72\end{array}$ & $\begin{array}{c}1.44(0.95-2.20) \\
.09\end{array}$ \\
\hline $\begin{array}{l}\text { Congenital } \\
\text { comorbidity }\end{array}$ & $\begin{array}{c}1.17(0.91-1.49) \\
.22\end{array}$ & $\begin{array}{c}1.43(0.82-2.49) \\
.21\end{array}$ & $\begin{array}{c}1.39(1.01-1.91) \\
.05\end{array}$ & $\begin{array}{c}1.19(0.92-1.55) \\
.19\end{array}$ & $\begin{array}{c}1.35(0.71-2.54) \\
.36\end{array}$ & $\begin{array}{c}1.46(1.03-2.07) \\
.03\end{array}$ \\
\hline Severity of illness & $\begin{array}{l}2.17(1.64-2.88) \\
\quad<.001\end{array}$ & $\begin{array}{l}6.67(3.96-11.25) \\
\quad<.001\end{array}$ & $\begin{array}{c}3.54(2.52-4.98) \\
\quad<.001\end{array}$ & $\begin{array}{c}1.28(0.93-1.76) \\
.13\end{array}$ & $\begin{array}{c}3.39(1.74-6.61) \\
<.001\end{array}$ & $\begin{array}{c}1.62(1.07-2.44) \\
.02\end{array}$ \\
\hline Premature & $\begin{array}{c}0.92(0.64-1.33) \\
.67\end{array}$ & $\begin{array}{c}1.20(0.54-2.67) \\
.65\end{array}$ & $\begin{array}{c}1.63(1.08-2.45) \\
.02\end{array}$ & $\begin{array}{c}0.80(0.54-1.19) \\
.28\end{array}$ & $\begin{array}{c}1.11(0.46-2.68) \\
.82\end{array}$ & $\begin{array}{c}1.54(0.97-2.43) \\
.07\end{array}$ \\
\hline Down syndrome & $\begin{array}{c}1.12(0.79-1.60) \\
.52\end{array}$ & $\begin{array}{c}0.90(0.36-2.28) \\
.83\end{array}$ & $\begin{array}{c}0.97(0.58-1.62) \\
.91\end{array}$ & - & - & - \\
\hline $\begin{array}{l}\text { Additional cardiac } \\
\quad \text { risk }\end{array}$ & $\begin{array}{c}1.29(0.90-1.86) \\
.17\end{array}$ & $\begin{array}{c}2.02(0.95-4.29) \\
.07\end{array}$ & $\begin{array}{c}1.63(1.02-2.60) \\
.04\end{array}$ & $\begin{array}{c}1.26(0.86-1.86) \\
.24\end{array}$ & $\begin{array}{c}1.79(0.75-4.30) \\
.19\end{array}$ & $\begin{array}{c}1.61(0.94-2.76) \\
.08\end{array}$ \\
\hline \multicolumn{7}{|l|}{$\begin{array}{l}\text { Procedure Reparative/ } \\
\text { corrective (ref) }\end{array}$} \\
\hline Palliative/staged & $\begin{array}{c}2.50(1.93-3.22) \\
\quad<.001\end{array}$ & $\begin{array}{c}0.86(0.40-1.86) \\
.71\end{array}$ & $\begin{array}{c}2.36(1.64-3.39) \\
<.001\end{array}$ & $\begin{array}{c}1.87(1.25-2.80) \\
\quad<.01\end{array}$ & $\begin{array}{c}0.71(0.20-2.54) \\
.60\end{array}$ & $\begin{array}{c}1.43(0.77-2.67) \\
.26\end{array}$ \\
\hline Ungrouped & $\begin{array}{c}0.93(0.72-1.21) \\
.61\end{array}$ & $\begin{array}{c}0.77(0.42-1.41) \\
.40\end{array}$ & $\begin{array}{c}1.21(0.86-1.72) \\
.28\end{array}$ & $\begin{array}{c}0.95(0.72-1.25) \\
.72\end{array}$ & $\begin{array}{c}1.02(0.52-1.99) \\
.96\end{array}$ & $\begin{array}{c}1.32(0.89-1.97) \\
.17\end{array}$ \\
\hline \multicolumn{7}{|l|}{ No bypass (reference) } \\
\hline Up to $90 \mathrm{~min}$ & $\begin{array}{c}0.48(0.35-0.66) \\
<.001\end{array}$ & $\begin{array}{c}0.25(0.06-1.14) \\
.07\end{array}$ & $\begin{array}{c}0.58(0.35-0.97) \\
.04\end{array}$ & $\begin{array}{c}0.69(0.48-1.00) \\
.05\end{array}$ & $\begin{array}{c}0.43(0.10-1.90) \\
.26\end{array}$ & $\begin{array}{c}1.15(0.64-2.08) \\
.64\end{array}$ \\
\hline$>90$ min & $\begin{array}{c}1.39(1.04-1.85) \\
.02\end{array}$ & $\begin{array}{c}6.11(2.20-16.99) \\
<.01\end{array}$ & $\begin{aligned} 2.45 & (1.56-3.84) \\
& <.001\end{aligned}$ & $\begin{array}{c}1.76(1.24-2.50) \\
\quad<.01\end{array}$ & $\begin{aligned} 6.63 & (2.43-18.07) \\
& <.001\end{aligned}$ & $\begin{array}{l}3.38(1.95-5.84) \\
\quad<.001\end{array}$ \\
\hline
\end{tabular}

Cardiac diagnosis group (main preoperative diagnosis) (A) hypoplastic left heart syndrome, truncus arteriosus, pulmonary atresia intact septum, (B) functionally univentricular heart, pulmonary atresia ventricular septal defect, (C) transposition of the great arteries all types, interrupted aortic arch, totally anomalous pulmonary venous connection, (D) patent ductus arteriosus, tricuspid valve anomalies, acquired heart disease, complete atrioventricular septal defect, (E) tetralogy of Fallot, mitral valve anomalies, isolated aortic stenosis, aortic regurgitation, aortic arch obstruction, subaortic obstruction, ventricular septal defect, atrial septal defect. RR, Risk ratio; $C I$, confidence interval; ECLS, extracorporeal life support. 
2-category risk model (Table 3), but they enabled specific delineation of the risk for multiple morbidity and ECLS. Neonatal status was even more strongly linked to ECLS (RR, 7.9; 95\% CI, 2.94-18.94) and multiple morbidity (RR, 10.6; 95\% CI, 6.22-17.78) than it was to morbidity in general. Likewise, in the more complex cardiac diagnoses, especially groups A and B (encompassing hypoplastic left heart syndrome, pulmonary atresia, truncus arteriosus, and functionally univentricular heart), the RRs were 4.3 (95\% CI, 1.1-16.6) and 8.2 (95\% CI, 3.0-24.2) for ECLS and 3.5 (95\% CI, 1.1-7.2) and 4.5 (95\% CI, 2.4-8.5) for multiple morbidities, suggesting these were strongly linked to these outcomes. The next most important risk factor for both these outcomes was prolonged bypass time more than 90 minutes. In particular, this was associated with a 6.6-fold risk of ECLS, noting that this intraoperative measure may reflect unexpected findings or technical challenges at operation. Increased severity of illness (which includes preprocedure mechanical ventilation or shock) was associated with a 3.7-fold higher incidence of ECLS and a 1.7fold risk for multiple morbidities. However, we advise some caution in interpreting these results because the number of ECLS patients was relatively low and CIs are wide. Surgical procedure category and univentricular heart were not significant risk factors in this model.

\section{Secondary Outcomes}

There were 9 patients discharged alive for whom life status information at 30 days was unavailable. Life status at 6 months was unavailable for 7 patients; these patients are not included in the corresponding mortality results. Missing date information for 9 patients led to them being excluded from the length of stay analyses.

Of 2861 patients, $37(1.3 \%)$ died within 30 days and 89 $(3.1 \%)$ died within 6 months of their first procedure. Postoperative length of stay, 30-day survival, and 6-month survival are shown in Table 5 by individual morbidity type. Given that these were secondary outcomes, not subjected to detailed risk modeling and included for descriptive purposes, we do not present $P$ values with this table. Postoperative length of stay is depicted in Figure 2.

While acknowledging that we did not undertake detailed risk models for our secondary outcomes of survival 6 months after operation and patient length of stay (both

TABLE 5. Postoperative length of stay, 30-day survival, and 6-month survival by individual morbidity type

\begin{tabular}{|c|c|c|c|}
\hline Morbidity type & $\begin{array}{l}\text { Median length of postoperative } \\
\text { hospital stay in days (IQR) }\end{array}$ & $\begin{array}{l}\text { 30-d survival from first } \\
\text { procedure }(\mathbf{N}=\mathbf{2 8 5 2})\end{array}$ & $\begin{array}{c}\text { 6-mo survival from first } \\
\text { procedure }(N=2845)\end{array}$ \\
\hline No morbidity & $8 \mathrm{~d}(5,13)$ & $\begin{array}{c}2216 / 2219 \\
99.9 \%(99.6,100)\end{array}$ & $\begin{array}{c}2202 / 2217 \\
99.3 \%(98.9,99.6)\end{array}$ \\
\hline Any morbidity & $24 \mathrm{~d}(15,42)$ & $\begin{array}{c}599 / 633 \\
94.6 \%(92.6,96.3)\end{array}$ & $\begin{array}{c}554 / 628 \\
88.2 \%(85.4,90.6)\end{array}$ \\
\hline Single morbidities aggregated & $20 \mathrm{~d}(13,31)$ & $\begin{array}{c}379 / 384 \\
98.7 \%(97.0,99.6)\end{array}$ & $\begin{array}{c}365 / 381 \\
95.8 \%(93.3,97.6)\end{array}$ \\
\hline Multi-morbidity & $35 \mathrm{~d}(22,56)$ & $\begin{array}{c}181 / 192 \\
94.3 \%(90.0,97.1)\end{array}$ & $\begin{array}{c}158 / 190 \\
83.2 \%(77.1,88.2)\end{array}$ \\
\hline ECLS & $43 \mathrm{~d}(20,84)$ & $\begin{array}{c}39 / 57 \\
68.4 \%(54.8,80.1)\end{array}$ & $\begin{array}{c}31 / 57 \\
54.4 \%(40.7,67.6)\end{array}$ \\
\hline Acute neurologic event & $19 \mathrm{~d}(12,39)$ & $\begin{array}{c}12 / 13 \\
92.3 \%(64.0,99.8)\end{array}$ & $\begin{array}{c}12 / 13 \\
92.3 \%(64.0,99.8)\end{array}$ \\
\hline Unplanned reoperation & $22 \mathrm{~d}(14,33)$ & $\begin{array}{c}54 / 54 \\
100 \%(93.4,100)\end{array}$ & $\begin{array}{c}50 / 54 \\
92.6 \%(82.1,97.9)\end{array}$ \\
\hline Feeding problems (feed) & $20.5 \mathrm{~d}(12,36)$ & $\begin{array}{c}94 / 94 \\
100 \%(96.2,100)\end{array}$ & $\begin{array}{c}90 / 91 \\
98.9 \%(94.0,100)\end{array}$ \\
\hline Renal support (renal) & $17 \mathrm{~d}(14,26)$ & $\begin{array}{c}39 / 39 \\
100 \%(91.0,100)\end{array}$ & $\begin{array}{c}37 / 39 \\
94.9 \%(82.7,99.4)\end{array}$ \\
\hline Major adverse event & $16.5 \mathrm{~d}(8,25)$ & $\begin{array}{c}29 / 33 \\
87.9 \%(71.8,96.6)\end{array}$ & $\begin{array}{c}28 / 33 \\
84.9 \%(68.1,94.9)\end{array}$ \\
\hline Necrotizing enterocolitis & $24.5 \mathrm{~d}(18.5,49.5)$ & $\begin{array}{c}30 / 30 \\
100 \%(88.4,100)\end{array}$ & $\begin{array}{c}28 / 30 \\
93.3 \%(77.9,99.2)\end{array}$ \\
\hline Surgical infection & $20.5 \mathrm{~d}(11,28)$ & $\begin{array}{c}25 / 25 \\
100 \%(86.3,100)\end{array}$ & $\begin{array}{c}25 / 25 \\
100 \%(86.3,100)\end{array}$ \\
\hline Prolonged pleural effusion & $20 \mathrm{~d}(14,28)$ & $\begin{array}{c}96 / 96 \\
100 \%(96.2,100)\end{array}$ & $\begin{array}{c}95 / 96 \\
99.0 \%(94.3,100)\end{array}$ \\
\hline
\end{tabular}

$\overline{I Q R}$, Interquartile range; $E C L S$, extracorporeal life support. 


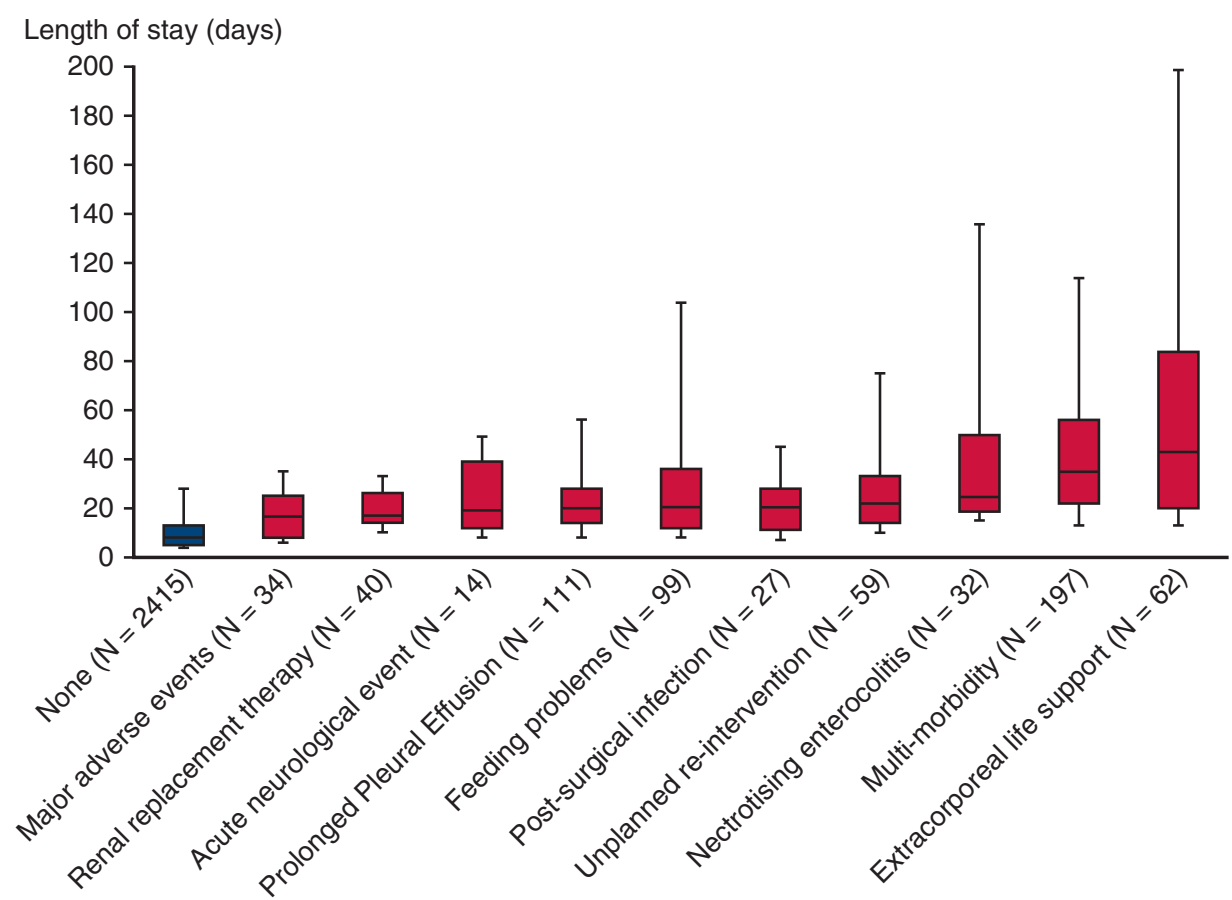

FIGURE 2. Postoperative length of stay by morbidity type. The boxplot shows the postoperative length of stay in days, for no selected morbidities (in blue), for each of the selected morbidities in isolation, multiple morbidities, and ECLS (all in red). The middle heavy bar represents the median, the box represents the IQR 25th (Q1) to 75th centiles (Q3), and the outer lines ending in a bar represent the range.

are linked to case complexity), we note that these measures were strongly associated with morbidity. The 6-month survival was significantly higher in those who had no selected morbidity at $99.3 \%(95 \%$ CI, 98.9-99.6) than those who had "any morbidity" at $88.2 \%$ (95\% CI, 85.4-90.6; $P<.001)$. Patients with any single selected morbidity, ECLS, or multi-morbidity had a significantly lower survival at 6 months compared with those with no selected morbidity $(P<.001)$. All morbidity groups had a significantly longer length of stay than patients with no selected morbidity.

\section{DISCUSSION}

This unique, large prospective multicenter study of the incidence of important early morbidities after pediatric cardiac surgery highlights some important points. Among 3090 procedures, $21.8 \%$ led to at least 1 of the selected morbidities. Of these 3090 procedures, $6.4 \%$ led to multiple morbidities and a further $2 \%$ led to ECLS, which in particular may be considered a near-miss adverse event. The most common of our included morbidities, all with rates greater than $5 \%$, were prolonged pleural effusion, feeding problems, and unplanned reoperation.

The patients who had none of the selected morbidities had shorter lengths of stay than those with 1 or more of the selected morbidities and were more likely to survive to 6 months. Although patients with 1 or more selected morbidity were more complex, the large differences we report in length of stay and survival at 6 months with morbidity emphasize the importance of these events for patients and families, and as potential future metrics for benchmarking.

The most important risk factors for the selected morbidities, as has been shown in previous studies ${ }^{24-26}$ included nonmodifiable risk factors of young age and more complex cardiac diseases. In addition, children who deteriorated before surgery requiring intensive care supports with severity of illness factors, which may in some instances be modifiable, were more likely to experience morbidity. Prolonged cardiopulmonary bypass time, which again in some cases be modifiable, was particularly associated with the key outcomes of ECLS and multiple morbidities.

Our study complements the findings of a number of initiatives that illustrate growing attention worldwide on the issue of surgical morbidity in this population, although a notable difference in our study was the selection of a list of morbidities incorporating perspectives from families and clinicians working outside specialist centers. ${ }^{9}$ Moreover, our stated remit was to include morbidities that were considered important based on prevalence and impact, rather than an exhaustive list of every morbidity. We note other important initiatives including activities of the Society of Thoracic Surgeons Taskforce Subcommittee on Patient Safety, which defined a range of unwanted events that may contribute to postoperative morbidity, including complications, adverse events, harm, medical error or injury, 
and near misses. ${ }^{27}$ Investigators used Society of Thoracic Surgeons Registry data to develop a composite scores to measure morbidity. ${ }^{25} \mathrm{~A}$ concern with this approach is that specific morbidities that may be amenable to quality improvement are less visible.

Prospective efforts include a Canadian study, which indicated that prospective monitoring of complications may lead to greater case ascertainment and thus a perception of higher complication rates. ${ }^{24}$ The Pediatric Cardiac Critical Care Consortium ( $\left.\mathrm{PC}^{4}\right)$ set up in 2009, with the aim to improve the quality of care to patients with critical pediatric and congenital cardiovascular disease in North America and abroad, provides partner sites who participate on a voluntary basis with access to contemporary data for quality improvement. $^{28,29}$

A motivation for our study was that although routine audit of postoperative mortality in pediatric cardiac surgery is well established in the United Kingdom via the NCHDA, ${ }^{11}$ stakeholders, including children's heart surgery programs, congenital heart patient support groups, and the national audit, want to add morbidity outcomes to the current reporting of mortality. In 2015 at the start of our study, the NCHDA initiated the capture of preliminary morbidity measurements based on our study protocols, but these outcomes have yet to be analyzed. The collection of morbidities by the NCHDA will over time enable a future registry-based study involving larger numbers of patients, which might enable a method of risk adjustment and national audit to be developed for routine use.

\section{Study Limitations}

We included only risk factors for morbidity that were available within mandatory and validated national audit data. Although this means that centers can analyze their ongoing case-mix with respect to these factors, we acknowledge that there may be other risk factors that we have not identified by taking this approach. It was necessary to collapse the risk factors of cardiac diagnosis and cardiac procedure into broad groups for our risk factor analyses, thus limiting interpretation of our results when considering specific individual conditions or procedures. Of note, we took an approach of categorizing cardiac procedures into 3 broad groups and cardiac diagnosis into 5 groups. We think cardiac diagnosis is an important factor to consider in outcome analyses because we recognize that the most complex patients may undergo a series of operations. Although we undertook extensive quality checks on our study data, no such processes are perfect. We found low rates for certain morbidities as stand-alone events, and the small numbers limited the risk factor analysis we were able to undertake for these individual stand-alone morbidities, for example, we did not have sufficient numbers to analysis incidence by specific procedure.

\section{CONCLUSIONS}

Our prospective multicenter study from the United Kingdom complements the international efforts in this important area. To assist with audit and quality assurance initiatives, we have developed software for local monitoring of complication rates in the United Kingdom, and we have co-developed information resources related to these findings for parents who report on rates of the selected morbidities, which will be available to UKbased clinicians to use during the surgical consent process. In the future, it is hoped that routine collection of important morbidity measures will complement the collection of mortality data by the national congenital heart diseases audit in the United Kingdom.

\section{Webcast}

You can watch a Webcast of this AATS meeting presentation by going to: https://aats.blob.core.windows.net/ media/19\%20AM/Tuesday_May7/202BD/202BD/S109\% 20-\%20Cardiopulmonary \%20Bypass $\% 20$ strategies/S109_ 7_webcast_113446908.mp4.

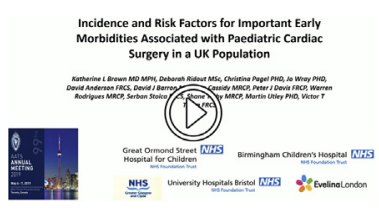

\section{Conflict of Interest Statement}

Authors have nothing to disclose with regard to commercial support.

Study contributors and advisors included Andrew Maclean, Sheryl Snowball, Luke Maidment, Sarah Bohannon, Liz Smith, Alison Jones, Kate Penny-Thomas, Joanne Webb, Sinead Cummins, John Stickley, Natasha Khan, Teresa Dickson, Ray Samson, Isobel Mcleod, Paul Wellman, Thomas Witter, Rhian Lakhani, Karen Sheehan, Kathleen Selway, Carrie Cherrington, Andrew Parry, Rob Tulloh, Bill Gaynor, Rodney Franklin, Lisa Allera, Kate Bull, Trevor Ritchens, Branko Mimic, Jon Smith, Lyvonne Tume, Vibeke Hjortdal, Michael Vath, Tom Treasure, Anne Keatley Clarke, Bea Tuten, and all the patients who participated in the study.

\section{References}

1. O'Brien SM, Clarke DR, Jacobs JP, Jacobs ML, Lacour-Gayet FG, Pizarro C, et al. An empirically based tool for analyzing mortality associated with congenital heart surgery. J Thorac Cardiovasc Surg. 2009;138: 1139-53.

2. Jenkins KJ, Gauvreau K, Newburger JW, Spray TL, Moller JH, Iezzoni LI. Consensus-based method for risk adjustment for surgery for congenital heart disease. J Thorac Cardiovasc Surg. 2002;123:110-8.

3. Lacour-Gayet F, Clarke D, Jacobs J, Gaynor W, Hamilton L, Jacobs M, et al. The Aristotle score for congenital heart surgery. Semin Thorac Cardiovasc Surg Pediatr Card Surg Annu. 2004; 7:185-91.

4. Brown KL, Ridout DA, Goldman AP, Hoskote A, Penny DJ. Risk factors for long intensive care unit stay after cardiopulmonary bypass in children. Crit Care Med. 2003;31:28-33. 
5. Costello JM, Cooper DS, Jacobs JP, Chai PJ, Kirsch R, Rosenthal T, et al. Intermediate-term outcomes after paediatric cardiac extracorporeal membrane oxygenation-what is known (and unknown). Cardiol Young. 2011;21(Suppl 2):118-23.

6. Newburger JW, Sleeper LA, Bellinger DC, Goldberg CS, Tabbutt S, Lu M, et al. Early developmental outcome in children with hypoplastic left heart syndrome and related anomalies: the single ventricle reconstruction trial. Circulation. 2012;125:2081-91.

7. Brown KL, Wray J, Wood TL, Mc Mahon AM, Burch M, Cairns J. Cost utility evaluation of extracorporeal membrane oxygenation as a bridge to transplant for children with end-stage heart failure due to dilated cardiomyopathy. J Heart Lung Transplant. 2009;28:32-8.

8. National Health Service. Safe and sustainable: children's congenital cardiac services 2011. Available at: http://www.specialisedservices.nhs.uk/safe sustainable/childrens-congenital-cardiac-services. Accessed March 31, 2012.

9. Pagel C, Brown KL, McLeod I, Jepps H, Wray J, Chigaru L, et al. Selection by a panel of clinicians and family representatives of important early morbidities associated with paediatric cardiac surgery suitable for routine monitoring using the nominal group technique and a robust voting process. BMJ Open. 2017;7: e014743.

10. Brown KL, Pagel C, Brimmell R, Bull K, Davis P, Franklin RC, et al. Definition of important early morbidities related to paediatric cardiac surgery. Cardiol Young. 2017;27:747-56.

11. NICOR. NICOR: National Institute for Cardiovascular Outcomes Research: Congenital Heart Diseases. London UK: University College London; 2017. Available at: www.nicor4.nicor.org.uk/CHD. Accessed December 20, 2018.

12. Rogers L, Brown KL, Franklin RC, Ambler G, Anderson D, Barron DJ, et al. Improving risk adjustment for mortality after pediatric cardiac surgery: the UK PRAiS2 Model. Ann Thorac Surg. 2017;104:211-9.

13. Crowe S, Brown KL, Pagel C, Muthialu N, Cunningham D, Gibbs J, et al. Development of a diagnosis- and procedure-based risk model for 30-day outcome after pediatric cardiac surgery. J Thorac Cardiovasc Surg. 2013; $145: 1270-8$.

14. Jacobs JP, O'Brien SM, Pasquali SK, Gaynor JW, Mayer JE Jr, Karamlou T, et al. The Society of Thoracic Surgeons congenital heart surgery database mortality risk model: part 2-clinical application. Ann Thorac Surg. 2015; 100:1063-70

15. Cole TJ, Freeman JV, Preece MA. British 1990 growth reference centiles for weight, height, body mass index and head circumference fitted by maximum penalized likelihood. Stat Med. 1998;17:407-29.

16. Brown KL, Rogers L, Barron DJ, Tsang V, Anderson D, Tibby S, et al. Incorporating comorbidity within risk adjustment for UK pediatric cardiac surgery. Ann Thorac Surg. 2017;104:220-6.

17. Aharon AS, Drinkwater DC Jr, Churchwell KB, Quisling SV, Reddy VS, Taylor M, et al. Extracorporeal membrane oxygenation in children after repair of congenital cardiac lesions. Ann Thorac Surg. 2001;72: 2095-102.

18. Alsoufi B, Shen I, Karamlou T, Giacomuzzi C, Burch G, Silberbach M, et al. Extracorporeal life support in neonates, infants, and children after repair of congenital heart disease: modern era results in a single institution. Ann Thorac Surg. 2005;80:15-21.

19. Beiras-Fernandez A, Deutsch M-A, Kainzinger S, Kaczmarek I, Sodian R, Ueberfuhr P, et al. Extracorporeal membrane oxygenation in 108 patients with low cardiac output - a single-center experience. Int J Artif Organs. 2011;34: 365-73.

20. Chauhan S, Malik M, Malik V, Chauhan Y, Kiran U, Bisoi AK. Extracorporeal membrane oxygenation after pediatric cardiac surgery: a 10 year experience. Ann Card Anaesth. 2011;14:19-24

21. Coskun KO, Coskun ST, Popov AF, Hinz J, El-Arousy M, Schmitto JD, et al. Extracorporeal life support in pediatric cardiac dysfunction. J Cardiothorac Surg. 2010;5:112.

22. Rubin DB. Multiple Imputation for Nonresponse in Surveys. New York: Wiley; 1987.

23. Stata Statistical Software: Release 14. College Station, TX: StataCorp LP; 2015.

24. Belliveau D, Burton HJ, O'Blenes SB, Warren AE, Hancock Friesen CL. Realtime complication monitoring in pediatric cardiac surgery. Ann Thorac Surg. 2012:94:1596-602.

25. Jacobs ML, O’Brien SM, Jacobs JP, Mavroudis C, Lacour-Gayet F, Pasquali SK, et al. An empirically based tool for analyzing morbidity associated with operations for congenital heart disease. J Thorac Cardiovasc Surg. 2013;145:1046-57.
26. Hickey PA, Connor JA, Cherian KM, Jenkins K, Doherty K, Zhang H, et al. International quality improvement initiatives. Cardiol Young. 2017;27:S61-8

27. Jacobs JP, Benavidez OJ, Bacha EA, Walters HL, Jacobs ML. The nomenclature of safety and quality of care for patients with congenital cardiac disease: a report of the Society of Thoracic Surgeons congenital database taskforce subcommittee on patient safety. Cardiol Young. 2008; 18(Suppl 2):81-91.

28. Gaies M, Werho DK, Zhang W, Donohue JE, Tabbutt S, Ghanayem NS et al. Duration of Postoperative mechanical ventilation as a quality metric for pediatric cardiac surgical programs. Ann Thorac Surg. 2018; 105:615-21.

29. Buckley JR, Graham EM, Gaies M, Alten JA, Cooper DS, Costello JM, et al Clinical epidemiology and centre variation in chylothorax rates after cardiac surgery in children: a report from the pediatric cardiac critical care consortium. Cardiol Young. 2017;1-8.

Key Words: cardiac surgery, complications, morbidity, outcome, pediatrics

\section{Discussion}

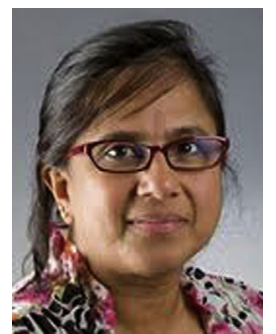

Dr Meena Nathan (Boston, Mass). You have involved multiple people to come up with your 9 important comorbidities, and it is important in future studies that key stakeholders be involved early in the process, and I congratulate you on doing that in a timely fashion.

I have several questions for you. In your morbidity analysis, you analyzed on the basis of procedures rather than at the patient level. How many of those 3090 procedures occurred during the same hospitalizations? Were there any that were counted twice during the same hospitalization?

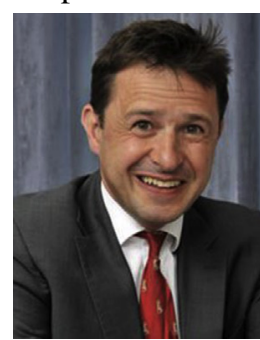

Dr David Barron (London, United Kingdom). They would have to have occurred during the hospital admission to be counted as a morbidity.

Dr Nathan. My question was, you had 2861 patients but 3090 operations.

Dr Barron. Procedures, yes.

Dr Nathan. How do you account for that?

Dr Barron. Of course, some of them were the same patients, such as a staged Norwood, they may have had their stage I and II during the period of the study so they would appear twice. If they had a reoperation during the same admission and if it was within 30 days, it would not count as a separate procedure. So you have to have 2 procedures at least 30 days apart for them to be entered.

Dr Nathan. Were there some that occurred during the same hospitalization?

Dr Barron. Yes. 
Dr Nathan. How do you account for which morbidity went with which procedure?

Dr Barron. It goes back to the primary procedure in that case.

Dr Nathan. You had a large sample size. I was wondering whether you considered additional subgroup analysis on the higher risk groups, such as neonates and maybe on palliative versus nonpalliative procedures?

Dr Barron. Yes, that is all in process now, so there is a lot of additional analysis that goes into particulars for each individual morbidity where we are studying the risk factors for each individual morbidity and how it is affecting survival.

Dr Nathan. And you did do a clustered analysis to account for center variation?

Dr Barron. Yes.

Dr Nathan. Did you consider analyzing volume of surgeon, volume of center as tertiles to look at variability?

Dr Barron. Exactly that, and we are doing it. There is surprisingly little variation between the centers, but the full analysis is still to come out. There are some real lessons. For instance, we don't understand why but in Birmingham we have a low incidence of need for renal support. So we are intrigued to look into these sort of things that the study has thrown up to see whether we can learn from each other and if there is anything differently that others can learn from.

Dr Nathan. Have you decided of these 9 which are the 5 you are going to prospectively monitor and audit at all your centers?

Dr Barron. It's the ones that are easiest to define and manage. So it's going to be need for renal support and for neurologic outcomes, a need for ECMO for unexpected reoperations during admission and for major adverse events.

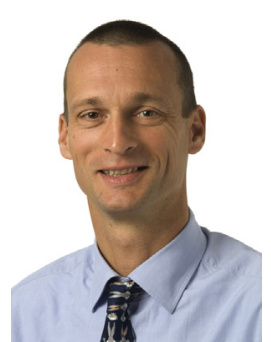

Dr Yves d'Udekem d'Acoz (Victoria, Australia). David, I think it's great work. It is nice to have this large set of data. I want to challenge you a bit and see how do you practically react to that? You have got your little app and what do you do from there? Do you go to your guys and say, hey, guys, your rate of stroke is high, your babies are not feeling well, you have to do something. And then there are some measurements that are not the same as the other ones, and I was particularly happy to look at the rate of unplanned reoperation, 5\%, which is what I estimate it should be. I am nervous when people tell me that they have a low rate of reoperation, because you want to ultimately have the patient get out of the hospital with a perfect operation even if it is at the cost of a reoperation.

Dr Barron. Very well said, good, you are quite right, we look at these things, but what are we going to do about it? I think for some of them they might at least focus everybody on the fact that things aren't going as well as you might think things are going. Things like infection rates, for sure, I think you can react to them and do something about it. For some of the others it may not necessarily be so easy, but at least it heightens your awareness, and I think at least you are armed a little bit with more information.

For reoperations, it's difficult to standardize for it because people's thresholds for reoperation will be different and you don't quite know, as you say, when it becomes a good thing or a bad thing. So we will be going into the whole analysis and looking at all the patients who had reoperations to try and make sort of an understanding of whether it reflected bad practice or whether it reflected good practice. 


\section{APPENDIX}

\section{Appendix E1. Definitions of Morbidity, From Brown} and Colleagues ${ }^{10}$

\begin{tabular}{|c|c|c|c|c|}
\hline Morbidity & Timescale for identification & Definition & $\begin{array}{l}\text { Measurement protocol (if } \\
\text { additional to definition) }\end{array}$ & $\begin{array}{c}\text { Minimum treatment } \\
\text { protocol }\end{array}$ \\
\hline $\begin{array}{l}\text { Acute neurologic } \\
\text { event }\end{array}$ & $\begin{array}{l}\text { Includes neurologic } \\
\text { morbidities that, based on } \\
\text { best clinical judgment, } \\
\text { arose as new findings } \\
\text { around the time of surgery } \\
\text { that were detected within } \\
\text { the same hospitalization as } \\
\text { the surgery. It is recognized } \\
\text { that in certain } \\
\text { circumstances such as } \\
\text { when a child is very sick on } \\
\text { life support, preprocedure } \\
\text { assessment is challenging, } \\
\text { in these circumstances as } \\
\text { full an evaluation as } \\
\text { possible to be completed, } \\
\text { incorporating serial } \\
\text { assessments over time. }\end{array}$ & $\begin{array}{l}\text { Neurologic events, including } \\
\text { seizure, abnormal } \\
\text { movement (includes } \\
\text { choreiform or athetoid), } \\
\text { focal neurologic deficit } \\
\text { (includes hemiplegia and } \\
\text { monoplegia), intracranial } \\
\text { hemorrhage, stroke, brain } \\
\text { death, reversible ischemic } \\
\text { neurologic dysfunction, } \\
\text { hypoxic ischemic } \\
\text { encephalopathy, spinal cord } \\
\text { ischemia, basal ganglia } \\
\text { damage, or brain stem } \\
\text { injury (includes abnormal } \\
\text { cough or gag reflex). }{ }^{\mathrm{E} 1}\end{array}$ & $\begin{array}{l}\text { Includes new abnormality in } \\
\text { any of the following: } \\
\text { - Electroencephalogram } \\
\text { - Brain scan (computed } \\
\text { tomography or } \\
\text { magnetic resonance) } \\
\text { - Clinical evaluation } \\
\text { (seizures or movement } \\
\text { disorder, focal } \\
\text { neurologic signs, } \\
\text { generalized neurologic } \\
\text { signs, altered conscious } \\
\text { level including even } \\
\text { brain death) }\end{array}$ & $\begin{array}{l}\text { The treatment protocol is } \\
\text { variable depending on the } \\
\text { type of neuro-morbidity. } \\
\text { Specialist consultation with a } \\
\text { neurologist, a full } \\
\text { evaluation of any brain } \\
\text { injury, and } \\
\text { neurodevelopmental } \\
\text { follow-up would be a } \\
\text { minimum. }\end{array}$ \\
\hline $\begin{array}{l}\text { Unplanned } \\
\text { reoperation or } \\
\text { reintervention }\end{array}$ & $\begin{array}{l}\text { Unplanned reinterventions are } \\
\text { procedures outside the } \\
\text { expected patient pathway, } \\
\text { which may be undertaken } \\
\text { at any time from the start of } \\
\text { the postoperative admission } \\
\text { up until } 30 \mathrm{~d} \text { after the } \\
\text { primary operation. } \\
\text { Additional procedures or } \\
\text { revisions undertaken within } \\
\text { the primary trip to the } \\
\text { operating theatre } \\
\text { (incorporating return onto } \\
\text { cardiopulmonary bypass) } \\
\text { are not included in the } \\
\text { definition of reoperation. }\end{array}$ & $\begin{array}{l}\text { Unplanned reinterventions } \\
\text { include procedures that } \\
\text { were not intended during } \\
\text { the planning phase, follow } \\
\text { an initial primary cardiac } \\
\text { surgery, and result in } \\
\text { "substantive alteration to } \\
\text { heart" incorporating } \\
\text { cardiac bypass, cardiac } \\
\text { nonbypass, pacemaker } \\
\text { placement, interventional } \\
\text { catheterizations, and } \\
\text { diaphragm plications } \\
\text { (which are not related to the } \\
\text { heart itself). The definition } \\
\text { does not include support or } \\
\text { other noncardiac surgery } \\
\text { procedures. }\end{array}$ & $\begin{array}{l}\text { Unplanned return to the } \\
\text { operating room or cardiac } \\
\text { catheter laboratory within } \\
30 \mathrm{~d} \text { (excludes } \\
\text { interventional catheters that } \\
\text { were planned } \\
\text { preoperatively; excluding } \\
\text { delayed chest closure, } \\
\text { excluding procedures for } \\
\text { bleeding) } \\
\text { (Includes diaphragm plication } \\
\text { and insertion of pacemaker } \\
\text { for surgically acquired } \\
\text { arrhythmia). }\end{array}$ & $\begin{array}{l}\text { Not applicable. The minimal } \\
\text { assessment is } \\
\text { cardiovascular evaluation } \\
\text { of the repair with } \\
\text { echocardiography and } \\
\text { tolerance of weaning from } \\
\text { life supports. }\end{array}$ \\
\hline $\begin{array}{l}\text { Feeding } \\
\text { problems }\end{array}$ & $\begin{array}{l}\text { A diagnosis of postoperative } \\
\text { feeding problems should be } \\
\text { considered during recovery } \\
\text { after surgery and before } \\
\text { discharge from the } \\
\text { specialist center to home or } \\
\text { to secondary care if the } \\
\text { child is unable to feed } \\
\text { normally. The goal is } \\
\text { detection of feeding } \\
\text { problems which are new } \\
\text { postsurgery, and it is } \\
\text { recognized that this may be } \\
\text { challenging where a child }\end{array}$ & 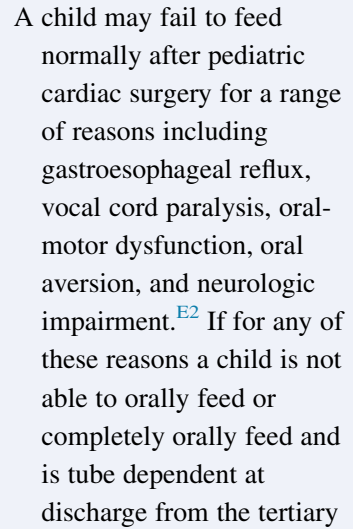 & $\begin{array}{l}\text { The requirement for any } \\
\text { feeding support. } \\
\text { Includes via the intravenous } \\
\text { route or via an enteral tube. } \\
\text { Excludes feeding support that } \\
\text { was present to treat a } \\
\text { primary problem diagnosed } \\
\text { before the surgery, feeding } \\
\text { support related to an } \\
\text { episode of necrotizing } \\
\text { enterocolitis, and feeding } \\
\text { support because the child } \\
\text { dislikes a special diet. }\end{array}$ & $\begin{array}{l}\text { Treatment includes } \\
\text { assessment by the dietician, } \\
\text { speech and language } \\
\text { therapist, and of the } \\
\text { patient's weight. Progress } \\
\text { with feeding should be } \\
\text { monitored by the clinical } \\
\text { care team responsible at } \\
\text { each stage of the journey. }\end{array}$ \\
\hline
\end{tabular}




\begin{tabular}{|c|c|c|c|c|}
\hline Morbidity & Timescale for identification & Definition & $\begin{array}{l}\text { Measurement protocol (if } \\
\text { additional to definition) }\end{array}$ & $\begin{array}{l}\text { Minimum treatment } \\
\text { protocol }\end{array}$ \\
\hline & $\begin{array}{l}\text { was not fed preoperatively } \\
\text { for cardiac reasons because } \\
\text { feeding ability will not have } \\
\text { been assessed objectively. }\end{array}$ & $\begin{array}{l}\text { center or at } 30 \mathrm{~d} \text { (if he or she } \\
\text { is otherwise clinically } \\
\text { stable enough to feed at that } \\
\text { time point), then a } \\
\text { postoperative feeding } \\
\text { problem will be diagnosed. }\end{array}$ & & \\
\hline $\begin{array}{l}\text { Need for renal } \\
\text { replacement } \\
\text { therapy }\end{array}$ & $\begin{array}{l}\text { Includes renal replacement } \\
\text { therapy when initiated as a } \\
\text { new support at any time } \\
\text { from the start of the } \\
\text { postoperative admission to } \\
\text { ICU up to } 30 \mathrm{~d} \text { after the } \\
\text { primary operation. }\end{array}$ & $\begin{array}{l}\text { The child requires renal } \\
\text { replacement therapy } \\
\text { (peritoneal dialysis or } \\
\text { hemofiltration) for renal } \\
\text { failure (oligo-anuria } \\
\text { of }<0.5 \mathrm{~mL} / \mathrm{kg} / \mathrm{h} \text { and } \\
\text { elevated creatinine level for } \\
\text { age) or fluid overload. In } \\
\text { patients in whom renal } \\
\text { support is required } \\
\text { alongside ECLS, the } \\
\text { primary morbidity is } \\
\text { viewed as ECLS. }\end{array}$ & $\begin{array}{l}\text { The measurement protocol is } \\
\text { simply the presence of } \\
\text { (new) renal support. } \\
\text { (Excludes renal support on } \\
\text { ECLS.) Data on renal } \\
\text { biochemistry and urine } \\
\text { output will be collected. }\end{array}$ & $\begin{array}{l}\text { Instigation of effective renal } \\
\text { replacement therapy. } \\
\text { If recovery of kidney function } \\
\text { does not occur within } 3 \text { to } \\
5 \text { wk then consultation with } \\
\text { pediatric renal physician is } \\
\text { required. }\end{array}$ \\
\hline $\begin{array}{l}\text { Major adverse } \\
\text { cardiac events } \\
\text { or never events }\end{array}$ & $\begin{array}{l}\text { Events within this morbidity } \\
\text { may be identified during } \\
\text { the tertiary hospital stay } \\
\text { (ward or ICU) after the } \\
\text { primary surgery. }\end{array}$ & $\begin{array}{l}\text { This morbidity includes: } \\
\text { - Cardiac arrest, where } \\
\text { the child receives any } \\
\text { chest compressions or } \\
\text { defibrillation. } \\
\text { - Chest reopening on the } \\
\text { ICU or ward for any } \\
\text { reason. } \\
\text { - Major hemorrhage in } \\
\text { the ICU after surgery. } \\
\text { - A 'Never Event' } \\
\text { applicable to pediatric } \\
\text { cardiac surgery as } \\
\text { selected from the } \\
\text { 'Never Events' list } \\
\text { published for National } \\
\text { Health Service for } \\
\text { 2015 }{ }^{\mathrm{E} 3} \text { (Including } \\
\text { wrong site or wrong } \\
\text { patient surgery, wrong } \\
\text { prosthesis surgery, } \\
\text { retained foreign object } \\
\text { post procedure, wrong } \\
\text { route administration of } \\
\text { medication, transfusion } \\
\text { or transplantation of } \\
\text { main red cell group } \\
\text { incompatible blood } \\
\text { components or organs, } \\
\text { misplaced nasogastric } \\
\text { or orogastric tubes, }\end{array}$ & $\begin{array}{l}\text { Major hemorrhage is defined } \\
\text { as bleeding > } 10 \mathrm{~mL} / \mathrm{kg} / \mathrm{h} \\
\text { on ICU for } 2 \text { consecutive } \\
\text { hours. } \\
\text { A 'Never Event' includes the } \\
\text { events listed plus harm to } \\
\text { the patient, for example, if a } \\
\text { nasogastric tube is } \\
\text { misplaced, detected and } \\
\text { removed in a timely manner } \\
\text { before any harm is done } \\
\text { then this is not a 'Never } \\
\text { Event.' Conversely, if the } \\
\text { misplaced nasogastric tube } \\
\text { is not noted, and feed is } \\
\text { given into the bronchus, } \\
\text { then this is a 'Never Event.' }\end{array}$ & $\begin{array}{l}\text { All events will result in } \\
\text { immediate treatment as part } \\
\text { of current practice. }\end{array}$ \\
\hline
\end{tabular}




\begin{tabular}{|c|c|c|c|c|}
\hline Morbidity & Timescale for identification & Definition & $\begin{array}{l}\text { Measurement protocol (if } \\
\text { additional to definition) }\end{array}$ & $\begin{array}{c}\text { Minimum treatment } \\
\text { protocol }\end{array}$ \\
\hline & & $\begin{array}{l}\text { - Tissue injury to limb or } \\
\text { vital organ such as } \\
\text { perforated viscus or } \\
\text { ischemic limb injury. }\end{array}$ & & \\
\hline ECLS & $\begin{array}{l}\text { ECLS after surgery and before } \\
\text { discharge from the tertiary } \\
\text { hospital, including the rare } \\
\text { cases when a child was on } \\
\text { ECLS before surgery. }\end{array}$ & $\begin{array}{l}\text { This morbidity is defined by } \\
\text { the presence of an ECLS } \\
\text { system connected to the } \\
\text { patient after the operation, } \\
\text { whether it was placed in the } \\
\text { operating theatre or in the } \\
\text { intensive care unit, and } \\
\text { whether the indication was } \\
\text { cardiac arrest, low cardiac } \\
\text { output state, poor cardiac } \\
\text { function, arrhythmia, } \\
\text { residual or recurrent } \\
\text { cardiac lesion, pulmonary } \\
\text { including pulmonary } \\
\text { hypertension or sepsis. }\end{array}$ & $\begin{array}{l}\text { It is recognized that children } \\
\text { on ECLS after pediatric } \\
\text { cardiac surgery have high } \\
\text { rates of other } \\
\text { complications, including } \\
\text { renal support, bleeding, } \\
\text { sepsis, sternal reopening, } \\
\text { and cardiac arrest. }{ }^{E} \text { Where } \\
\text { such complications arise as } \\
\text { part of ECLS, the morbidity } \\
\text { is defined as ECLS. }\end{array}$ & $\begin{array}{l}\text { The morbidity is in fact a } \\
\text { treatment modality offered } \\
\text { so this is not applicable. } \\
\text { Centers offering ECLS } \\
\text { follow protocols based on } \\
\text { those provided by the } \\
\text { ECLS organization. }\end{array}$ \\
\hline $\begin{array}{l}\text { Necrotizing } \\
\text { enterocolitis }\end{array}$ & $\begin{array}{l}\text { Necrotizing enterocolitis as a } \\
\text { new diagnosis from after } \\
\text { surgery until discharge } \\
\text { from the tertiary hospital. }\end{array}$ & $\begin{array}{l}\text { Necrotizing enterocolitis class } \\
\text { 1a or } 1 \mathrm{~b},{ }^{\text {E5 }} \text { which } \\
\text { incorporates babies with } \\
\text { systemic signs of } \\
\text { inflammation and } \\
\text { abdominal clinical signs } \\
\text { such as distension or larger } \\
\text { than normal gastric } \\
\text { aspirates or mild rectal } \\
\text { bleeding but no radiologic } \\
\text { changes are included, if a } \\
\text { general surgery specialist } \\
\text { has seen the child and } \\
\text { commenced a course of } \\
\text { intravenous antibiotics and } \\
\text { parenteral nutrition for } 5 \text { to } \\
7 \text { d. Cases of severe } \\
\text { necrotizing enterocolitis } \\
\text { with radiologic signs } \\
\text { systemic instability and } \\
\text { bowel perforation are also } \\
\text { included. }\end{array}$ & $\begin{array}{l}\text { Data in respect of systemic } \\
\text { clinical signs, intestinal } \\
\text { signs and radiology will be } \\
\text { collected, as well as the } \\
\text { treatments deployed, thus } \\
\text { enabling the necrotizing } \\
\text { enterocolitis diagnosis to be } \\
\text { graded between 1a and } 3 \mathrm{~b} \text {. }\end{array}$ & $\begin{array}{l}\text { Consultation with general } \\
\text { surgery and further } \\
\text { management in respect of } \\
\text { antibiotics, nutrition, } \\
\text { radiologic investigation, } \\
\text { and surgical intervention. }\end{array}$ \\
\hline $\begin{array}{l}\text { Surgical site } \\
\text { infection and } \\
\text { bloodstream } \\
\text { infection }\end{array}$ & $\begin{array}{l}\text { Surgical site and bloodstream } \\
\text { infections diagnosed within } \\
\text { the hospital admission after } \\
\text { surgery or after } \\
\text { readmission to the same } \\
\text { unit during postoperative } \\
\text { recovery, where the treating } \\
\text { clinical team assesses the } \\
\text { infection to be linked to the } \\
\text { recent operation. It is noted } \\
\text { that mediastinitis may be } \\
\text { detected more than } 30 \mathrm{~d}\end{array}$ & $\begin{array}{l}\text { Deep surgical site infection or } \\
\text { mediastinitis includes any } \\
\text { infection of an incised } \\
\text { wound that undergoes any } \\
\text { reintervention by a surgeon } \\
\text { (eg, opening of the wound, } \\
\text { vacuum dressing), } \\
\text { mediastinitis and false } \\
\text { aneurysm, independent of } \\
\text { culture positivity. } \\
\text { Bloodstream infection } \\
\text { includes both catheter }\end{array}$ & $\begin{array}{l}\text { Deep surgical site infection } \\
\text { excludes superficial site } \\
\text { infection managed without } \\
\text { a surgeon's reoperation by } \\
\text { conventional nurse dressing } \\
\text { only, even if the wound } \\
\text { heals by secondary } \\
\text { intention. }\end{array}$ & $\begin{array}{l}\text { The minimum treatment } \\
\text { protocol consists of } \\
\text { antibiotics based on } \\
\text { organism and sensitivities, } \\
\text { and where relevant the } \\
\text { removal of the line. } \\
\text { Surgical intervention may } \\
\text { be required for deep } \\
\text { surgical site and in some } \\
\text { cases of endocarditis. Both } \\
\text { conditions require }\end{array}$ \\
\hline
\end{tabular}




\begin{tabular}{|c|c|c|c|c|}
\hline Morbidity & Timescale for identification & Definition & $\begin{array}{l}\text { Measurement protocol (if } \\
\text { additional to definition) }\end{array}$ & $\begin{array}{l}\text { Minimum treatment } \\
\text { protocol }\end{array}$ \\
\hline & $\begin{array}{l}\text { after cardiac surgery, }{ }^{\mathrm{E} 6} \text { thus } \\
\text { this time cutoff is not } \\
\text { applicable. }\end{array}$ & $\begin{array}{l}\text { related and noncatheter } \\
\text { related. Cases have } \\
\text { systemic signs of infection, } \\
\text { a positive culture not } \\
\text { judged to be a contaminant, } \\
\text { and in the case of line } \\
\text { related a catheter in place } \\
\text { with positive cultures from } \\
\text { the line or from the line tip } \\
\text { when removed. } \\
\text { Endocarditis based on } \\
\text { clinical, imaging, or culture } \\
\text { evidence judged to be } \\
\text { diagnostic of endothelial/ } \\
\text { endocardial infection and } \\
\text { its sequelae cardiac or } \\
\text { extra-cardiac. }\end{array}$ & & $\begin{array}{l}\text { prolonged antibiotic } \\
\text { therapy. }\end{array}$ \\
\hline $\begin{array}{l}\text { Prolonged pleural } \\
\text { effusion or } \\
\text { chylothorax }\end{array}$ & $\begin{array}{l}\text { Prolonged pleural effusion is a } \\
\text { postprocedural effusion } \\
\text { with duration }>10 \mathrm{~d} \text {. } \\
\text { Chylothorax is diagnosed } \\
\text { from after surgery until } \\
\text { discharge from the tertiary } \\
\text { hospital. }\end{array}$ & $\begin{array}{l}\text { Either a chylous pleural } \\
\text { effusion or significant } \\
\text { chylous pericardial } \\
\text { effusion or significant } \\
\text { chylous ascites or a } \\
\text { prolonged nonchylous } \\
\text { effusion that necessitates } \\
\text { thoracic drainage at least } \\
10 \mathrm{~d} \text { after index cardiac } \\
\text { surgery. }\end{array}$ & $\begin{array}{l}\text { Chylous effusions are } \\
\text { characterized by milky } \\
\text { appearance and a pleural } \\
\text { fluid white blood cell count } \\
\text { of greater than } 1000 \text { cells/ } \\
\mu \mathrm{L} \text { with lymphocytes } \\
\text { greater than } 80 \%{ }^{\mathrm{E} 7} \text { If the } \\
\text { child is on normal feeds } \\
\text { the triglyceride level in the } \\
\text { pleural fluid will } \\
\text { be }>1.1 \text { mmol/L or the } \\
\text { ratio between the pleural } \\
\text { triglyceride level and the } \\
\text { serum triglyceride level } \\
\text { will exceed } 1 .\end{array}$ & $\begin{array}{l}\text { Diet consisting of medium } \\
\text { chain triglycerides or low } \\
\text { fat for chylothorax. On a } \\
\text { patient-by-patient basis } \\
\text { other treatments include } \\
\text { parenteral nutrition, } \\
\text { octreotide infusion, } \\
\text { intervention for venous } \\
\text { obstruction thoracic duct } \\
\text { ligation, and pleuradhesis. }\end{array}$ \\
\hline
\end{tabular}

$E C L S$, Extracorporeal life support; $I C U$, intensive care unit.

\section{E-References}

E1. Dominguez TE, Wernovsky G, Gaynor JW. Cause and prevention of central nervous system injury in neonates undergoing cardiac surgery. Semin Thorac Cardiovasc Surg. 2007; 19:269-77.

E2. Medoff-Cooper B, Naim M, Torowicz D, Mott A. Feeding, growth, and nutrition in children with congenitally malformed hearts. Cardiol Young. 2010;20(Suppl 3): 149-53.

E3. National Health Service. Never-events. In: England N, ed. Patient Safety. London: National Health Service England; 2015. Patient Safety Guideline.

E4. Chaturvedi RR, Macrae D, Brown KL, Schindler M, Smith EC, Davis KB, et al. Cardiac ECMO for biventricular hearts after paediatric open heart surgery. Heart. 2004;90:545-51.
E5. McElhinney DB, Hedrick HL, Bush DM, Pereira GR, Stafford PW, Gaynor JW, et al. Necrotizing enterocolitis in neonates with congenital heart disease: risk factors and outcomes. Pediatrics. 2000;106: 1080-7.

E6. Sohn AH, Schwartz JM, Yang KY, Jarvis WR, Guglielmo BJ, Weintrub PS. Risk factors and risk adjustment for surgical site infections in pediatric cardiothoracic surgery patients. Am J Infect Control. 2010;38: 706-10.

E7. Zuluaga MT. Chylothorax after surgery for congenital heart disease. Curr Opin Pediatr. 2012;24:291-4. 


\section{Appendix E2. Cardiac Procedure Groups Based on Rogers and Colleagues ${ }^{12}$}

(A) Palliative or staged

(B) reparative or corrective

(C) ungrouped or

Specific procedure groups

ambiguous

\begin{tabular}{ll}
\hline Group 1 & \\
Norwood procedure (stage 1) & A \\
HLHS hybrid approach & A \\
Group 2 & \\
TAPVC repair + arterial shunt & A \\
Truncus and interruption repair & B \\
Truncus arteriosus repair & B \\
Interrupted aortic arch repair & B \\
Arterial switch + aortic arch & B \\
obstruction repair (with or & \\
without VSD closure) &
\end{tabular}

Group 3

Arterial shunt

A

Group 4

Repair of total anomalous

B

pulmonary venous connection

Arterial switch + VSD closure

Isolated pulmonary artery band

B

A

Group 5

PDA ligation (surgical)

C

Group 6

Arterial switch (for isolated

B

transposition)

Isolated coarctation/hypoplastic B

aortic arch repair

Aortopulmonary window repair

B

Group 7

Senning or Mustard procedure

Ross-Konno procedure

A

Mitral valve replacemen

Pulmonary vein stenosis procedure A

Pulmonary atresia VSD repair

Tetralogy with absent pulmonary valve repair

Unifocalization procedure (with/ without shunt)

Group 8

Heart transplant

Tricuspid valve replacement

Aortic valve repair

Pulmonary valve replacement

Aortic root replacement (not Ross)

Cardiac conduit replacement

Continued

(A) Palliative or staged

(B) reparative or corrective

(C) ungrouped or

Specific procedure groups ambiguous

Isolated RV to PA conduit

construction

Tricuspid valve repair

A

Group 9

Multiple VSD closure B

Atrioventricular septal defect and B tetralogy repair

Cor triatriatum repair

Supravalvar aortic stenosis repair

Rastelli - REV procedure

\section{C}

B

B

B

Group 10

Bidirectional cavopulmonary shunt

A

Group 11

Atrioventricular septal defect

B

(complete) repair

Group 12

Fontan procedure

A

Group 13

Aortic valve replacement - Ross B

Subvalvar aortic stenosis repair B

Mitral valve repair B

Sinus venosus ASD or PAPVC B

repair

Group 14

Atrioventricular septal defect B

(partial) repair

Tetralogy and Fallot-type DORV B

repair

Vascular ring procedure

B

Group 15

Anomalous coronary artery repair B

Aortic valve replacement - non- $\quad$ B

Ross

ASD repair $\quad B$

VSD repair B

No specific procedure group

No specific procedure

$\mathrm{C}$

$H L H S$, Hypoplastic left heart syndrome; TAPVC, total anomalous pulmonary venous connection; $V S D$, ventricular septal defect; $P D A$, patent ductus arteriosus closure; $R V$, right ventricle; $P A$, pulmonary artery; $A S D$, atrial septal defect; $P A P V C$, partial anomalous pulmonary venous connection; $D O R V$, double-outlet right ventricle. 


\section{Appendix E2. Cardiac Diagnosis Groups Based on Rogers and Colleagues $^{12}$}

\begin{tabular}{|c|c|}
\hline Diagnosis groups & Group \\
\hline \multicolumn{2}{|l|}{ Group 1} \\
\hline HLHS & A \\
\hline Truncus arteriosus & A \\
\hline Pulmonary atresia and IVS & A \\
\hline \multicolumn{2}{|l|}{ Group 2} \\
\hline Functionally UVH & B \\
\hline Pulmonary atresia and VSD & B \\
\hline \multicolumn{2}{|l|}{ Group 3} \\
\hline TGA+VSD/DORV-TGA & $\mathrm{C}$ \\
\hline Interrupted aortic arch & $\mathrm{C}$ \\
\hline \multicolumn{2}{|l|}{ Group 4} \\
\hline PDA & $\mathrm{D}$ \\
\hline \multicolumn{2}{|l|}{ Group 5} \\
\hline Miscellaneous primary congenital diagnosis & $\mathrm{D}$ \\
\hline Tricuspid valve abnormality (including Ebstein's) & $\mathrm{D}$ \\
\hline TAPVC & $\mathrm{C}$ \\
\hline Procedure & N/A \\
\hline Comorbidity & N/A \\
\hline Normal & N/A \\
\hline Empty/unknown & N/A \\
\hline \multicolumn{2}{|l|}{ Group 6} \\
\hline Acquired & $\mathrm{D}$ \\
\hline \multicolumn{2}{|l|}{ Group 7} \\
\hline AVSD & $\mathrm{D}$ \\
\hline Fallot/DORV Fallot & $\mathrm{E}$ \\
\hline \multicolumn{2}{|l|}{ Group 8} \\
\hline Aortic valve stenosis (isolated) & $\mathrm{E}$ \\
\hline Mitral valve abnormality & E \\
\hline Miscellaneous congenital terms & $\mathrm{E}$ \\
\hline \multicolumn{2}{|l|}{ Group 9} \\
\hline TGA + IVS & $\mathrm{C}$ \\
\hline \multicolumn{2}{|l|}{ Group 10} \\
\hline Aortic arch obstruction + VSD/ASD & $\mathrm{E}$ \\
\hline Pulmonary stenosis & $\mathrm{E}$ \\
\hline \multicolumn{2}{|l|}{ Group 11} \\
\hline Subaortic stenosis (isolated) & $\mathrm{E}$ \\
\hline Aortic regurgitation & $\mathrm{E}$ \\
\hline VSD & $\mathrm{E}$ \\
\hline ASD & $\mathrm{E}$ \\
\hline Arrhythmia & $\mathrm{E}$ \\
\hline
\end{tabular}

$\overline{H L H S, \text { Hypoplastic left heart syndrome; IVS, intact ventricular septum; } U V H \text {, univen- }}$ tricular heart; $V S D$, ventricular septal defect; $D O R V$, double-outlet right ventricle; $T G A$, transposition of the great arteries; PDA, patent ductus arteriosus; TAPVC, totally anomolous pulmonary venous connection; $N / A$, not available; $A V S D$, atrioventricular septal defect; $A S D$, atrial septal defect. 


\section{Appendix E3. Case Mix and Volume by Center}

Center by age bands

\begin{tabular}{|c|c|c|c|c|}
\hline Hospital site & Neonate & Infant & Child & Total \\
\hline \multirow[t]{2}{*}{1.} & 120 & 444 & 415 & 979 \\
\hline & 12.26 & 45.35 & 42.39 & 100.00 \\
\hline \multirow[t]{2}{*}{2.} & 109 & 237 & 192 & 538 \\
\hline & 20.26 & 44.05 & 35.69 & 100.00 \\
\hline \multirow[t]{2}{*}{3.} & 130 & 259 & 337 & 726 \\
\hline & 17.91 & 35.67 & 46.42 & 100.00 \\
\hline \multirow[t]{2}{*}{4.} & 98 & 219 & 201 & 518 \\
\hline & 18.92 & 42.28 & 38.80 & 100.00 \\
\hline \multirow[t]{2}{*}{5.} & 71 & 132 & 126 & 329 \\
\hline & 21.58 & 40.12 & 38.30 & 100.00 \\
\hline \multirow[t]{2}{*}{ Total } & 528 & 1291 & 1271 & 3090 \\
\hline & 17.09 & 41.78 & 41.13 & 100.00 \\
\hline
\end{tabular}

Center by diagnosis category

\begin{tabular}{|c|c|c|c|c|c|c|}
\hline \multirow[b]{2}{*}{ Hospital site } & \multicolumn{5}{|c|}{ Cardiac diagnosis complexity } & \multirow[b]{2}{*}{ Total } \\
\hline & $\overline{\mathbf{A}}$ & $\overline{\mathbf{B}}$ & $\mathbf{C}$ & $\mathbf{D}$ & $\overline{\mathbf{E}}$ & \\
\hline \multirow[t]{2}{*}{1} & 58 & 101 & 78 & 340 & 402 & 979 \\
\hline & 5.92 & 10.32 & 7.97 & 34.73 & 41.06 & 100.00 \\
\hline \multirow[t]{2}{*}{2.} & 47 & 42 & 59 & 175 & 215 & 538 \\
\hline & 8.74 & 7.81 & 10.97 & 32.53 & 39.96 & 100.00 \\
\hline \multirow[t]{2}{*}{3.} & 124 & 110 & 69 & 206 & 217 & 726 \\
\hline & 17.08 & 15.15 & 9.50 & 28.37 & 29.89 & 100.00 \\
\hline \multirow[t]{2}{*}{4.} & 32 & 67 & 62 & 184 & 173 & 518 \\
\hline & 6.18 & 12.93 & 11.97 & 35.52 & 33.40 & 100.00 \\
\hline \multirow[t]{2}{*}{5.} & 16 & 21 & 56 & 118 & 118 & 329 \\
\hline & 4.86 & 6.38 & 17.02 & 35.87 & 35.87 & 100.00 \\
\hline \multirow[t]{2}{*}{ Total } & 277 & 341 & 324 & 1023 & 1125 & 3090 \\
\hline & 8.96 & 11.04 & 10.49 & 33.11 & 36.41 & 100.00 \\
\hline
\end{tabular}

Center by procedure

\begin{tabular}{|c|c|c|c|c|}
\hline \multirow[b]{2}{*}{ Hospital site } & \multicolumn{3}{|c|}{ Procedure category } & \multirow[b]{2}{*}{ Total } \\
\hline & A: Staged/palliative & B: Reparative/corrective & C: Ungrouped/ambiguous & \\
\hline \multirow[t]{2}{*}{1.} & 110 & 553 & 316 & 979 \\
\hline & 11.24 & 56.49 & 32.28 & 100.00 \\
\hline \multirow[t]{2}{*}{2.} & 72 & 323 & 143 & 538 \\
\hline & 13.38 & 60.04 & 26.58 & 100.00 \\
\hline \multirow[t]{2}{*}{3.} & 205 & 356 & 165 & 726 \\
\hline & 28.24 & 49.04 & 22.73 & 100.00 \\
\hline \multirow[t]{2}{*}{4.} & 86 & 266 & 166 & 518 \\
\hline & 16.60 & 51.35 & 32.05 & 100.00 \\
\hline \multirow[t]{2}{*}{5.} & 37 & 225 & 67 & 329 \\
\hline & 11.25 & 68.39 & 20.36 & 100.00 \\
\hline \multirow[t]{2}{*}{ Total } & 510 & 1723 & 857 & 3090 \\
\hline & 16.50 & 55.76 & 27.73 & 100.00 \\
\hline
\end{tabular}

